msh-mss Mathématiques et sciences humaines

163 | Automne 2003

Théorie du choix social : cinquantenaires

\title{
La mesure du pouvoir de vote
}

The measurement of voting power

Nicolas-Gabriel Andjiga, Fréderic Chantreuil et Dominique Lepelley

\section{OpenEdition}

Journals

Édition électronique

URL : http://journals.openedition.org/msh/2920

DOI : $10.4000 /$ msh. 2920

ISSN : 1950-6821

\section{Éditeur}

Centre d'analyse et de mathématique sociales de l'EHESS

\section{Édition imprimée}

Date de publication : 1 septembre 2003

ISSN : 0987-6936

\section{Référence électronique}

Nicolas-Gabriel Andjiga, Fréderic Chantreuil et Dominique Lepelley, «La mesure du pouvoir de vote », Mathématiques et sciences humaines [En ligne], 163 | Automne 2003, mis en ligne le 10 février 2006, consulté le 23 juillet 2020. URL : http://journals.openedition.org/msh/2920 ; DOI : https://doi.org/ $10.4000 / m s h .2920$ 


\title{
LA MESURE DU POUVOIR DE VOTE ${ }^{1}$
}

\author{
Nicolas-Gabriel ANDJIGA, ${ }^{2}$ Frédéric CHANTREUIL ${ }^{3}$ \\ Dominique LEPELLEY ${ }^{4}$
}

\begin{abstract}
RÉSUMÉ - Comment peut-on mesurer le pouvoir de vote des individus ou des groupes d'individus dans un processus de décision collective? L'objet principal de ce texte est de recenser les différentes réponses quantitatives qui ont été apportées à cette question. Divers «indices de pouvoir» sont ainsi présentés, analysés et comparés à l'aide d'une application au Conseil de l'Union européenne, puis en termes de propriétés normatives et d'interprétations probabilistes.
\end{abstract}

MOTS CLÉS - Indices de pouvoir, Pouvoir de vote, Jeux pondérés

SUMMARY - The Measurement of Voting Power

How can we measure the voting power of individuals or groups of individuals in a collective choice process? The aim of this study is to review the various numerical answers that have been given to this question. A number of power indices are introduced and compared, not only through an application to the European Union Council, but also in terms of normative properties and probabilistic interpretations.

KEYWORDS - Power Indices, Voting Power, Weighted Games

JEL Classification Number - D71

\section{INTRODUCTION}

De nombreuses institutions prennent leurs décisions à l'aide de procédures de vote dans lesquelles les différents intervenants ne disposent pas tous du même poids, c'està-dire du même nombre de votes. Les raisons qui justifient cette façon de procéder sont variées. Dans des organisations internationales comme l'Union européenne, il s'agit de représenter à peu près équitablement les populations de tailles différentes des pays membres. Le même type de motivation est à l'origine des différences dans

\footnotetext{
${ }^{1}$ Article reçu le 10 janvier 2003, révisé le 18 avril 2003, accepté le 05 mai 2003.

${ }^{2}$ École normale supérieure, B.P. 47, Yaoundé, Cameroun, andjiga@yahoo.fr

${ }^{3}$ INRA-ESR, 4, allée Bobierre, CS 61103, 35011 Rennes cedex, chantreu@roazhon.inra.fr

${ }^{4}$ GEMMA, Maison de la recherche en sciences humaines, Université de Caen, Esplanade de la Paix, 14032 Caen cedex, dominique.lepelley@econ.unicaen.fr
} 
le nombre de délégués attribués à chaque commune dans les institutions intercommunales. On peut aussi allouer les votes en tenant compte des contributions financières de chacun des participants à la décision collective, comme dans les assemblées générales d'actionnaires, de co-propriétaires ou bien encore dans les institutions monétaires internationales. Enfin, les différences de poids peuvent être le résultat d'un processus démocratique : dans les assemblées politiques, le poids de chaque parti correspond au nombre de sièges qu'il détient.

Quelles que soient les raisons conduisant à l'attribution de poids différents aux différents électeurs, l'idée sous-jacente est toujours la même : il s'agit de donner plus de pouvoir de décision à certains intervenants dans le processus de choix collectif. Intuitivement, un votant est d'autant plus puissant que son nombre de votes, son poids, est élevé et l'on peut être tenté de considérer que le pouvoir de vote d'un électeur est proportionnel à son poids. Par exemple (voir [Leech, 2000]), les ÉtatsUnis, qui possèdent $18 \%$ du total des votes au Fonds monétaire international et à la Banque mondiale, sont souvent présentés comme disposant de $18 \%$ du pouvoir de vote. D'une manière générale, cette façon de voir les choses est manifestement erronée. Il est facile de s'en convaincre. Considérons pour cela l'exemple suivant, emprunté à [Demange, 2001]. Soit une assemblée tripartite constituée d'un petit parti disposant de $6 \%$ des sièges et de deux grands partis réunissant chacun $47 \%$ des sièges. Si les décisions sont prises à la majorité (simple) des votes, alors le petit parti possède a priori autant de pouvoir que les autres, puisqu'il a la même capacité que les deux autres à former une majorité. Si, en revanche, le seuil de décision est fixé à $2 / 3$, le petit parti perd tout pouvoir puisqu'une décision ne peut être prise qu'avec l'accord des deux grands partis. La réalité du pouvoir de décision, entendu comme la capacité d'influencer l'issue d'un vote, dépend ainsi d'une part de la répartition complète des poids (la donnée du seul poids d'un électeur ne suffit pas à déterminer son pouvoir) et d'autre part de la règle de vote.

Comment peut-on alors rendre compte, numériquement, de la réalité des rapports de force dans un processus de décision collective du type de ceux que nous venons d'évoquer? Les indices de pouvoir constituent des tentatives de réponse à cette question. L'intérêt de ces indices est double. En premier lieu, ils sont susceptibles (de manière plus ou moins convaincante, comme nous le verrons) d'analyser la répartition du pouvoir de vote entre les différentes parties prenantes d'un processus donné de décision collective. Ainsi ces indices ont-ils été intensivement utilisés pour étudier le pouvoir au sein de différents parlements nationaux ou au sein d'institutions internationales, compte tenu de la règle de vote existante. En second lieu, ils permettent d'évaluer les conséquences de changements dans les règles de décision sur le pouvoir des différents membres d'une assemblée. Cette utilisation prospective peut aider à choisir les mécanismes vérifiant certains principes d'équité (on peut par exemple souhaiter que le pouvoir des intervenants soit proportionnel à leur poids). Les indices peuvent donc être utilisés à des fins positives et descriptives mais aussi dans un but normatif. 
Historiquement, le premier indice de pouvoir défini de manière formelle est celui de Shapley et Shubik $[1954]^{5}$. Pendant une vingtaine d'années, les débats se sont essentiellement concentrés sur l'analyse et la comparaison de cet indice et de son concurrent « intime », apparu quelques années plus tard et traditionnellement attribué à Banzhaf [1965]. Au cours des vingt-cinq années suivantes (particulièrement au cours des dix dernières), la littérature sur les indices s'est considérablement développée et renouvelée. D'une part, de nouveaux indices sont apparus; d'autre part, la diversité des approches et des auteurs s'est accrue, enrichissant ainsi le débat comme en témoignent les deux ouvrages récents de Felsenthal et Machover [1998] et de Holler et Owen [2001]. Ce renouveau a été particulièrement marqué en Europe ${ }^{6}$ où le principal organe de décision de l'Union, le Conseil des ministres, utilise une règle de vote pondéré : les problèmes de repondération posés par les élargissements successifs de 1995 et de 2004 ont suscité un nombre impressionnant d'articles et ont ravivé l'intérêt des scientifiques, sinon des politiques, pour l'analyse du pouvoir de vote.

L'objet de la présente étude est d'une part de recenser les principaux indices proposés dans la littérature, et d'autre part de présenter les différentes approches qui permettent d'analyser et de comparer ces indices ${ }^{7}$. Nous espérons ainsi contribuer à éclairer le lecteur francophone sur un domaine particulièrement actif de la recherche en théorie du vote ${ }^{8}$.

L'article est structuré de la manière suivante. La Section 2 présente quelques définitions ainsi que les principales notations que nous utiliserons tout au long de l'étude. La section suivante introduit, dans l'ordre chronologique de leur apparition dans la littérature, diverses mesures possibles du pouvoir de vote. Nous appliquons ensuite ces différentes mesures à l'analyse de la répartition du pouvoir de vote au sein du Conseil de l'Union européenne (Section 4). Cette application met en évidence le caractère possiblement divergent des conclusions obtenues et pose le problème du choix du « bon » indice. La Section 5 expose les démarches consistant à caractériser axiomatiquement les indices de pouvoir ou, plus simplement, à déterminer les propriétés normatives que vérifient les mesures concurrentes. L'intérêt et les limites de ces approches sont mis en évidence. Nous présentons dans la Section 6 une démarche d'une nature différente, qui a connu un certain renouveau au cours de ces dernières

\footnotetext{
${ }^{5} \mathrm{Ce}$ constat ne doit pas faire oublier les travaux précurseurs de Martin (voir [Riker, 1986] et, surtout, de Penrose [1946]). Le lecteur intéressé par ces aspects historiques pourra se référer utilement aux récentes contributions de Falck [2002] et Felsenthal et Machover [2002].

${ }^{6}$ Falck [2002] considère, à juste titre nous semble-t-il, que le centre de gravité de la recherche (théorique et empirique) sur les indices de pouvoir s'est déplacé au cours des dernières années des États-Unis vers l'Europe.

${ }^{7}$ Les travaux de synthèse les plus récents sur l'analyse (quantitative) du pouvoir de vote sont dus à Laruelle [1998] et Felsenthal et Machover [1998]. De très nombreux articles ont paru ou circulé depuis 1998. Nous rendons compte de certains d'entre eux dans les pages qui suivent.

${ }^{8}$ Les références en français sur les indices de pouvoir sont extrêmement rares. Les deux premiers articles écrits dans cette langue sont, à notre connaissance, ceux de Junn [1972] et de Monjardet [1972], parus dans Mathématiques et Sciences humaines et traitant du pouvoir au sein du Conseil de Sécurité de l'ONU. Des références plus récentes sont, outre Falck [2002], celles de Bobay [2001], Bonnet et Lepelley [2001] et Caulier [2001] qui, toutes, traitent d'applications des indices de pouvoir.
} 
années : il s'agit d'associer aux différents indices, autant que faire se peut, des interprétations probabilistes susceptibles de les éclairer d'un jour nouveau et d'aider l'utilisateur à choisir la mesure pertinente. Les principales contributions relevant de cette démarche sont brièvement exposées. La section finale présente les débats qui agitent actuellement la recherche relative aux indices de pouvoir et évoque certaines approches alternatives récentes.

\section{NOTATIONS ET DÉFINITIONS}

L'étude du pouvoir de vote s'est, pour une large part, développée en étroite connexion avec la théorie des jeux coopératifs. C'est donc à l'aide de notions et d'outils issus de cette théorie que nous présenterons, dans la section suivante, les indices de pouvoir. L'objet de cette section est de préciser les notations que nous allons utiliser et d'introduire les éléments de théorie des jeux qui nous seront nécessaires.

Un jeu de contrôle (ou jeu de vote) est un couple $(N, v)$, où $N=\{1, \ldots, i, \ldots, n\}$ est l'ensemble des « joueur » et $v$ une fonction qui associe à tout sous-ensemble de $N$ une valeur dans $\{0,1\}$.

Nous noterons en lettre majuscule les ensembles et en lettre minuscule correspondante le nombre d'éléments de ces ensembles. Ainsi une coalition $S$ est un sousensemble non vide de $N$ et nous noterons par $s$ le nombre de joueurs contenus dans $S$.

Une coalition $S$ est gagnante si $v(S)=1$ et perdante si $v(S)=0$. Nous noterons par $\mathcal{G}(v)$ (respectivement $\mathcal{G}_{i}(v)$ ) l'ensemble des coalitions gagnantes (respectivement l'ensemble des coalitions gagnantes contenant le joueur $i$ ).

Si nous considérons l'ensemble des joueurs comme un ensemble d'individus prenant part à un processus de décision collective, nous pouvons en général modéliser ce processus par un jeu de contrôle. Une coalition gagnante est alors définie comme un ensemble de votants tels que s'ils votent à l'unanimité pour une proposition, cette dernière est acceptée; on dit aussi que cette coalition est une majorité généralisée. La fonction $v$ précise donc le statut de chaque coalition et par conséquent formalise le processus de décision collective. Soulignons que l'abstention est ignorée dans le modèle : face à une proposition, un joueur ne peut que voter « oui » ou «non $\gg^{9}$

Un joueur $i$ est décisif pour une coalition gagnante $S$ si la défection de ce joueur rend la coalition $S$ perdante, c'est-à-dire si $v(S)=1$ et $v(S-\{i\})=0$. Nous noterons par $d(S)$ le nombre de joueurs décisifs dans la coalition $S$, et $\mathcal{D}_{i}(v)$ l'ensemble des coalitions pour lesquelles le joueur $i$ est décisif.

Une coalition minimale gagnante est une coalition gagnante qui ne contient que des joueurs décisifs. Nous noterons par $\mathcal{M}(v)$ (respectivement $\mathcal{M}_{i}(v)$ ) l'ensemble des coalitions minimales gagnantes (respectivement l'ensemble des coalitions minimales gagnantes contenant le joueur $i$ ).

Lorsque aucune ambiguïté n'est possible, les ensembles $\mathcal{G}(v), \mathcal{G}_{i}(v), \mathcal{D}_{i}(v), \mathcal{M}(v)$ et $\mathcal{M}_{i}(v)$ seront simplement notés $\mathcal{G}, \mathcal{G}_{i}, \mathcal{D}_{i}, \mathcal{M}$ et $\mathcal{M}_{i}$.

\footnotetext{
${ }^{9}$ Des travaux récents (voir [Felsenthal et Machover, 1998]) s'efforcent de dépasser cette restriction de l'analyse en considérant des jeux « ternaires » autorisant la prise en compte de l'abstention.
} 
Un jeu simple est un jeu de contrôle monotone, c'est-à-dire que $v(S) \leq v(T)$ pour tout $S \subseteq T$, et tel que la fonction $v$ ne soit pas identiquement nulle.

Un jeu simple est qualifié de pondéré s'il existe un quota $q$ et un poids $w_{i}$ positif pour chaque joueur tel que :

$$
v(S)= \begin{cases}1 & \text { si } \sum_{i \in S} w_{i} \geq q \\ 0 & \text { autrement. }\end{cases}
$$

Un jeu pondéré sera noté $v:\left[q ; w_{1}, w_{2}, \ldots, w_{n}\right]$. Certains des indices que nous allons étudier s'appliquent spécifiquement à cette classe de jeux. C'est la raison pour laquelle celle-ci sera privilégiée dans les analyses qui vont suivre.

Un jeu simple est propre si le complémentaire d'une coalition gagnante est toujours perdante $: S \in \mathcal{G}(v) \Rightarrow N-S \notin \mathcal{G}(v)$.

Un jeu simple est fort si le complémentaire d'une coalition perdante est toujours gagnante $: S \notin \mathcal{G}(v) \Rightarrow N-S \in \mathcal{G}(v)$.

On notera que si la première de ces conditions est peu contraignante, la seconde est beaucoup moins universelle. Ainsi, un jeu pondéré est propre si $q>w / 2$, avec $w=\sum_{i \in N} w_{i}$; un jeu pondéré est fort si ( $w$ étant supposé impair) $q=(w+1) / 2$. Nous conclurons cette section en introduisant le jeu simple pondéré suivant, qui nous servira dans la Section 3 à illustrer les indices présentés : $v:[3 ; 2,1,1,1]$. Ce jeu comporte quatre joueurs, que nous noterons $a, b, c$ et $d$. On peut constater qu'il est propre et fort. On vérifie par ailleurs que la seule coalition gagnante ne contenant pas le joueur $a$ est celle qui contient les trois autres joueurs. De même la seule coalition perdante contenant le joueur $a$ est celle où il est un singleton. Les autres coalitions perdantes sont celles qui ne contiennent pas le joueur $a$ et de cardinal inférieur ou égal à 2 .

Les tableaux suivants, que nous utiliserons pour les calculs des différents indices de pouvoir, donnent la liste des coalitions gagnantes (Tableau 1) et des coalitions minimales gagnantes (Tableau 2). Dans ces tableaux, un 1 à l'intersection de la ligne figurant la coalition $S$ et de la colonne correspondant au joueur $i$ signifie que le joueur $i$ est décisif dans la coalition $S$; un 0 signifie que $i$ n'est pas décisif. L'avantdernière colonne donne le nombre de joueurs décisifs dans la coalition et la dernière colonne le nombre de joueurs dans la coalition.

Tableau 1

\begin{tabular}{|c|c|c|c|c|c|c|}
\hline Coalitions gagnantes & Joueur $a$ & Joueur $b$ & Joueur $c$ & Joueur $d$ & $d(S)$ & $s$ \\
\hline$a, b$ & 1 & 1 & 0 & 0 & 2 & 2 \\
\hline$a, c$ & 1 & 0 & 1 & 0 & 2 & 2 \\
\hline$a, d$ & 1 & 0 & 0 & 1 & 2 & 2 \\
\hline$a, b, c$ & 1 & 0 & 0 & 0 & 1 & 3 \\
\hline$a, b, d$ & 1 & 0 & 0 & 0 & 1 & 3 \\
\hline$a, c, d$ & 1 & 0 & 0 & 0 & 1 & 3 \\
\hline$b, c, d$ & 0 & 1 & 1 & 1 & 3 & 3 \\
\hline$a, b, c, d$ & 0 & 0 & 0 & 0 & 0 & 4 \\
\hline
\end{tabular}


Tableau 2

\begin{tabular}{|c|c|c|c|c|c|c|}
\hline Coalitions minimales gagnantes & Joueur $a$ & Joueur $b$ & Joueur $c$ & Joueur $d$ & $d(S)$ & $s$ \\
\hline$a, b$ & 1 & 1 & 0 & 0 & 2 & 2 \\
\hline$a, c$ & 1 & 0 & 1 & 0 & 2 & 2 \\
\hline$a, d$ & 1 & 0 & 0 & 1 & 2 & 2 \\
\hline$b, c, d$ & 0 & 1 & 1 & 1 & 3 & 3 \\
\hline
\end{tabular}

\section{INDICES DE POUVOIR}

La liste des indices que nous allons brièvement décrire et illustrer ne vise pas à l'exhausivité ${ }^{10}$ Certaines mesures ne sont pas présentées car nous les avons considérées comme trop proches d'indices présents dans notre liste ${ }^{11}$. Nous avons d'autre part ignoré les indices qui, à la suite de Rae [1969], mesurent la satisfaction plutôt que le pouvoir des votants. Le recensement proposé inclut cependant, outre tous les indices aujourd'hui considérés comme classiques, deux indices qui n'apparaissent dans aucune autre liste $\mathrm{e}^{12}$.

\subsection{INDICE DE Shapley-ShubiK}

Le premier indice de pouvoir que nous considérons est celui de Shapley-Shubik [1954]. À l'origine, la valeur de Shapley [1953] est un concept de théorie des jeux construit pour être appliqué aux jeux sous forme de fonction caractéristique. Shapley et Shubik ont considéré son application à la classe des jeux simples et l'ont proposé comme une mesure de pouvoir a priori dans un processus de vote. La justification qu'ils en donnent est la suivante. Considérons un groupe d'individus votant l'amendement d'une loi selon la procédure suivante :

a) ils votent chacun leur tour ;

b) dès qu'une majorité est atteinte, l'individu qui a voté en dernier reçoit une « unité de pouvoir » puisque l'amendement est passé grâce à sa voix. Notons que cet individu est décisif dans la coalition formée par lui-même et tous ceux qui le précèdent.

Si nous supposons que l'ordre dans lequel les individus prennent part au vote est déterminé de manière aléatoire et équiprobable, nous pouvons déterminer le nombre moyen de fois où un individu donné est décisif. Pour tout jeu simple $(N, v)$, l'indice de Shapley-Shubik d'un joueur $i$ est ce nombre. Il est donné par :

$$
S S_{i}(v)=\sum_{S \subseteq N, S \ni i} \frac{(s-1) !(n-s) !}{n !}[v(S)-v(S-\{i\})] .
$$

\footnotetext{
${ }^{10} \mathrm{~A}$ notre connaissance, la liste d'indices la plus fournie que l'on puisse trouver est celle que proposent Pajala et alii [2002] sur le site internet POWERSLAVE.

${ }^{11}$ C'est le cas des indices de Coleman [1986], qui sont proches dans leur principe de l'indice (normalisé) de Banzhaf.

${ }^{12}$ Il s'agit de l'indice d'Andjiga-Berg [1996], Berg [1999] et de celui de Chakravarty [2000].
} 
Cette expression revient à diviser le nombre de permutations des joueurs pour lesquelles le joueur $i$ est décisif par le nombre total de permutations possibles. Comme toute permutation comprend un et un seul joueur décisif, il est clair que l'on a $\sum_{i} S S_{i}(v)=1$. L'indice de Shapley-Shubik est donc, par définition, normalisé.

En appliquant le principe de calcul de cet indice à notre exemple, on obtient, à l'aide du Tableau 1, les résultats suivants :

$$
S S_{a}(v)=1 / 2 \text { et } S S_{b}(v)=S S_{c}(v)=S S_{d}(v)=1 / 6 .
$$

\subsection{INDICES DE BANZHAF}

Un indice quelque peu différent a été proposé par Banzhaf [1965]. Son objectif était d'aider à résoudre certains débats juridiques concernant les normes d'équité constitutionnelle pour les systèmes de représentation électorale. Banzhaf considère, comme Shapley et Shubik, que la mesure du pouvoir du joueur $i$ doit dépendre du nombre de fois où il est décisif. Toutefois dans le processus de décision de Banzhaf le vote n'est pas séquentiel : les coalitions votent en bloc. Le «score » de Banzhaf du joueur $i$ est par conséquent le nombre de coalitions possibles (et non le nombre de permutations possibles) pour lesquelles $i$ est décisif.

Pour un jeu $(N, v)$, l'indice (normalisé) de Banzhaf du joueur $i$ est alors défini par :

$$
\tilde{B}_{i}(v)=\frac{d_{i}(v)}{\sum_{j=1}^{n} d_{j}(v)}
$$

avec $d_{i}(v)=\sum_{S \subseteq N, S \ni i}[v(S)-v(S-\{i\})]$; autrement dit, $d_{i}(v)$ représente le nombre de coalitions décisives contenant $i$.

Dubey et Shapley [1979] suggèrent une autre pondération du score de Banzhaf. Pour un jeu simple $(N, v)$, l'indice non normalisé de Banzhaf du joueur $i$ est donné par

$$
B_{i}(v)=\frac{d_{i}(v)}{2^{n-1}}=\frac{1}{2^{n-1}} \sum_{S \subseteq N, S \ni i}[v(S)-v(S-\{i\})] .
$$

Cette formulation de l'indice de Banzahf, que Dubey et Shapley justifient par des arguments probabilistes $\left(2^{n-1}\right.$ peut s'interpréter comme le nombre de coalitions dont le joueur $i$ fait partie), est la plus utilisée dans la littérature. Notons que l'indice normalisé de Banzhaf est souvent associé au nom de Coleman et que l'indice non normalisé trouve son origine dans les travaux de Penrose [1946].

On vérifie aisément, grâce au Tableau 1, que :

$$
\begin{aligned}
& B_{a}(v)=3 / 4 \text { et } B_{b}(v)=B_{c}(v)=B_{d}(v)=1 / 4, \\
& \tilde{B}_{a}(v)=1 / 2 \text { et } \tilde{B}_{b}(v)=\widetilde{B}_{c}(v)=\widetilde{B}_{d}(v)=1 / 6 .
\end{aligned}
$$

\subsection{INDICE DE JOHNSTON}

Johnston [1978] propose une modification de la pondération de l'indice de Banzhaf, en considérant que la mesure du pouvoir devrait dépendre du nombre de joueurs décisifs dans une coalition donnée. Son intuition est que le pouvoir d'un joueur décisif 
dans une coalition sera d'autant plus faible que le nombre de joueurs décisifs dans cette coalition sera élevé. Précisément, il suggère que le pouvoir attribuable à une coalition gagnante soit divisé également entre les différents votants décisifs de cette coalition. Dans un jeu simple $(N, v)$, le score de Johnston de $i$ est donc donné par :

$$
J_{i}(v)=\sum_{S \in \mathcal{D}_{i}(v)} \frac{1}{d(S)}[v(S)-v(S-\{i\})]
$$

$d(S)$ désignant le nombre de joueurs décisifs dans $S$. L'indice de Johnston du joueur $i$ est alors

$$
\tilde{J}_{i}(v)=\frac{J_{i}(v)}{\sum_{j \in N} J_{j}(v)} .
$$

Dans notre exemple, la répartition du pouvoir selon cet indice est la suivante (voir Tableau 1) :

$$
\tilde{J}_{a}(v)=9 / 14 \text { et } \tilde{J}_{b}(v)=\tilde{J}_{c}(v)=\tilde{J}_{d}(v)=5 / 42 .
$$

\subsection{Indice DE DEEGAN-PACKEL}

Deegan et Packel [1978] proposent un indice de pouvoir basé sur le principe de taille de Riker [1962]. Ce principe revient à considérer que seules les coalitions minimales gagnantes se forment. L'indice de Deegan-Packel est obtenu en supposant que les coalitions minimales gagnantes se forment de manière équiprobable et que chaque joueur d'une coalition minimale gagnante reçoit un «montant de pouvoir » inversement proportionnel à la taille de cette coalition. Pour un jeu simple $(N, v)$, l'indice de Deegan-Packel du joueur $i$ est ainsi donné par $(m(v)$ désignant le cardinal de $\mathcal{M}(v))$

$$
D P_{i}(v)=\frac{1}{m(v)} \sum_{S \in \mathcal{M}(v), S \ni i} \frac{1}{s}[v(S)-v(S-\{i\})]=\frac{1}{m(v)} \sum_{S \in \mathcal{M}_{i}(v)} \frac{1}{s} .
$$

On notera que $\sum_{i} D P_{i}(v)=1$. En appliquant ce principe de calcul à l'exemple, nous obtenons (voir Tableau 2) :

$$
D P_{a}(v)=3 / 8 \text {, et } D P_{b}(v)=D P_{c}(v)=D P_{d}(v)=5 / 24 \text {. }
$$

\subsection{INDICE DE HOLLER-PACKEL}

Holler et Packel [1983] proposent une modification de l'indice de Deegan-Packel en supposant que tous les joueurs d'une coalition minimale gagnante reçoivent le même montant de pouvoir, quelle que soit la taille de cette coalition. Il s'agit pour eux de redéfinir l'indice de Deegan-Packel de manière à prendre en compte les situations où la valeur associée à une coalition gagnante correspond, non à un bien privé divisible, mais à un bien collectif dont tous les membres de la coalition peuvent profiter sans 
exclusion ni rivalité ${ }^{13}$. Pour un jeu simple $(N, v)$, le score de Holler-Packel du joueur $i$ est :

$$
H P_{i}(v)=\frac{1}{m(v)} \sum_{S \in \mathcal{M}_{i}(v)}[v(S)-v(S-\{i\})]=\frac{m_{i}(v)}{m(v)} .
$$

L'indice de Holler-Packel du joueur $i$ est obtenu par la normalisation suivante :

$$
\tilde{H P} P_{i}(v)=\frac{H P_{i}(v)}{\sum_{j \in N} H P_{j}(v)}=\frac{m_{i}(v)}{\sum_{j \in N} m_{j}(v)} .
$$

La répartition du pouvoir qui en résulte dans l'exemple est la suivante (voir Tableau 2) :

$$
\tilde{H P} P_{a}(v)=1 / 3, \tilde{H P} P_{b}(v)=\tilde{H P}{ }_{c}(v)=\tilde{H P} P_{d}(v)=2 / 9 .
$$

Notons que les cinq indices précédents sont ceux que l'on peut aujourd'hui considérer comme classiques. Les analyses comparatives disponibles dans la littérature considèrent en général tout ou partie de cet ensemble de cinq indices.

\subsection{INDICES DE CURIEL}

Curiel [1987] propose un nouvel indice (en fait une famille d'indices) fondé(e), comme les deux indices précédents, sur le principe de taille de Riker. L'idée de Curiel est de relâcher l'hypothèse (implicite) d'équiprobabilité d'occurrence ou de formation des coalitions minimales gagnantes. À chaque coalition $S \subseteq N, S \neq \emptyset$, Curiel associe un poids $r_{S}>0$ représentant une mesure de la probabilité d'occurrence de la coalition $S$. Pour un ensemble de coalitions $\mathcal{C}$, nous notons $\sum_{S \in \mathcal{C}} r_{S}$ par $r(\mathcal{C})$. Pour tout jeu simple $(N, v)$ et toute coalition $S \in 2^{N}$, la probabilité d'occurrence de la coalition $S, p_{S}(v)$, est définie par :

$$
p_{S}(v)=\left\{\begin{array}{l}
0 \text { si } S \notin \mathcal{M}(v) \\
r_{S} / r(\mathcal{M}(v)) \text { si } S \in \mathcal{M}(v) .
\end{array}\right.
$$

Ainsi, pour toute coalition $S, p_{S}(v) \geq 0$ et $p(\mathcal{M}(v))=\sum_{S \in \mathcal{M}(v)} p_{S}(v)=1$. Le score de Curiel du joueur $i$ est donné par :

$$
\delta_{i}(v)=\sum_{S \in \mathcal{M}_{i}(v)} p_{S}(v)
$$

L'indice de Curiel est obtenu par la normalisation suivante :

$$
\tilde{\delta}_{i}(v)=\frac{\delta_{i}(v)}{\sum_{j=1}^{n} \delta_{j}(v)} .
$$

Il est clair que cet indice ne peut se calculer que si l'on connaît les poids $r_{S}$. La première distribution de poids suggérée par Curiel revient à supposer l'équiprobabilité d'occurrence des coalitions minimales gagnantes. Dans ce cas, l'indice de Curiel

\footnotetext{
${ }^{13}$ Pour cette raison, l'indice de Holler-Packel est également connu sous l'appellation de « Public Good Index » (PGI).
} 
coïncide avec celui de Holler-Packel. Une seconde distribution de poids consiste à supposer que la probabilité d'occurrence d'une coalition est inversement proportionnelle à sa taille. En d'autres termes, nous avons $r_{S}=|S|^{-1}$. Dans ce cas, l'indice de Curiel est identique à celui de Deegan-Packel.

\subsection{INDICE DE COLOMER-MarTinez}

Colomer [1996] et Colomer-Martinez [1995] proposent un indice de pouvoir également fondé sur le principe de taille de Riker. Se situant dans le cadre particulier de la formation d'une coalition gouvernementale, ils défendent l'idée selon laquelle un indice de pouvoir ne sert pas uniquement à estimer la capacité d'un parti à faire basculer le résultat d'un vote mais également à mesurer le pouvoir de ce parti au sein des coalitions auxquelles il appartient. Pour un jeu simple pondéré $v:\left[q ; w_{1}, \ldots, w_{n}\right]$, l'indice de Colomer-Martinez du joueur $i$ est défini par :

$$
C M_{i}(v)=\frac{\sum_{S \in \mathcal{M}_{i}(v)} w_{i}}{\sum_{S \in M} \sum_{j \in S} w_{j}}=\frac{w_{i} m_{i}(v)}{\sum_{j=1}^{n} w_{j} m_{j}(v)} .
$$

À l'aide du Tableau 2, on établit que cet indice conduit dans l'exemple à la répartition suivante :

$$
C M_{a}(v)=1 / 2 \text { et } C M_{b}(v)=C M_{c}(v)=C M_{d}(v)=1 / 6 .
$$

\subsection{INDICE D'ANDJIGA-BERG}

Andjiga [1996] et Berg [1999] proposent (indépendamment) un indice fondé sur les principes suivants :

(1) seules les coalitions gagnantes se forment et elles se forment de manière équiprobable;

(2) un joueur $i$ d'une coalition $S$ reçoit une fraction de pouvoir inversement proportionnelle à la taille de cette coalition $S$ lorsque le joueur $i$ est décisif dans $S$.

Le score d'Andjiga-Berg du joueur $i$ est donné par :

$$
A B_{i}(v)=\frac{1}{g(v)} \sum_{S \in \mathcal{G}_{i}(v)} \frac{1}{s}[v(S)-v(S-\{i\})],
$$

$g(v)$ désignant le nombre de coalitions gagnantes. L'indice d'Andjiga-Berg résulte de la normalisation suivante :

$$
\tilde{A B_{i}}(v)=\frac{A B_{i}(v)}{\sum_{j=1}^{n} A B_{j}(v)}
$$

On obtient dans l'exemple (voir Tableau 1) :

$$
\tilde{A B}_{a}(v)=1 / 2 \text { et } \tilde{A B_{b}}(v)=\tilde{A B_{c}}(v)=\tilde{A B_{d}}(v)=1 / 6 .
$$




\subsection{INDICE DE ChakRAVARTY}

Le dernier indice que nous considérons a été récemment proposé par Chakravarty [2000]. Chakravarty suggère que le pouvoir absolu d'un joueur $i$, dans le cadre des jeux simples pondérés, peut être mesuré par le nombre de fois où il est décisif, pondéré par son poids $w_{i}$. Le pouvoir d'un joueur est alors la fraction de pouvoir absolu total qu'il possède. On notera que la justification que donne l'auteur de son indice est essentiellement d'ordre théorique : il s'agit pour lui de proposer une sophistication de l'indice normalisé de Banzhaf $\tilde{B}_{i}(v)$ dotée de « bonnes » propriétés, que $\tilde{B}_{i}(v)$ ne possède pas ${ }^{14}$.

L'indice de Chakravarty du joueur $i$ dans le jeu $v:\left[q ; w_{1}, \ldots, w_{n}\right]$ est donné par :

$$
C_{i}(v)=\frac{w_{i} d_{i}(v)}{\sum_{j=1}^{n} w_{j} d_{j}(v)}
$$

L'application de ce principe de calcul conduit dans l'exemple aux résultats suivants :

$$
C_{a}(v)=2 / 3 \text { et } C_{b}(v)=C_{c}(v)=C_{d}(v)=1 / 9 .
$$

\subsection{UNE FORMULATION GÉNÉRALE}

La présentation de différents indices à laquelle nous venons de procéder suggère qu'ils sont tous construits selon les mêmes principes généraux. Chacun d'entre eux apparaît en effet comme une somme pondérée du type suivant :

$$
\alpha_{i}(v)=\sum_{S \in \mathcal{X}, S \ni i} f(i, S)[v(S)-v(S-\{i\})]
$$

où $\mathcal{X}$ désigne l'ensemble des coalitions (gagnantes) « admissibles » et $f(i, S)$ est une fonction « poids » qui dépend de la coalition $S$ et (dans certains cas) du joueur $i$ considéré.

Il s'agit donc essentiellement de compter le nombre total de coalitions admissibles pour lesquelles le joueur $i$ est décisif (tous les indices s'accordent sur ce point) en affectant une certaine pondération à chacune de ces coalitions. La somme ainsi obtenue peut alors éventuellement être normalisée, soit de manière à suggérer une interprétation probabiliste simple (comme nous le verrons dans la Section 6), soit pour faire en sorte que la somme des indices des différents joueurs soit égale à l'unité.

Construire un indice revient donc à répondre aux deux questions suivantes:

1) Quelles coalitions peut-on s'attendre à voir se former? (i.e. quelles sont les coalitions admissibles?)

2) Quel poids va-t-on donner à un joueur $i$ dans chacune des coalitions admissibles où le joueur considéré est décisif?

Les différents indices présentés répondent de la manière suivante à chacune de ces questions :

\footnotetext{
${ }^{14}$ Voir sur ce point la Section 5 .
} 
Tableau 3

\begin{tabular}{|c|c|c|}
\hline & Coalitions admissibles & Poids de $i$ dans $S$ \\
\hline Shapley-Shubik & coalitions gagnantes & $(s-1) !(n-s) ! / n !$ \\
\hline Banzhaf & coalitions gagnantes & 1 \\
\hline Johnston & coalitions gagnantes & $1 / d(S)$ \\
\hline Deegan-Packel & coalitions minimales gagnantes & $1 / s$ \\
\hline Holler-Packel & coalitions minimales gagnantes & 1 \\
\hline Colomer-Martinez & coalitions minimales gagnantes & $w_{i}$ \\
\hline Andjiga-Berg & coalitions gagnantes & $1 / s$ \\
\hline Chakravarty & coalitions gagnantes & $w_{i}$ \\
\hline
\end{tabular}

On retrouve alors les indices que nous avons introduits dans la Section précédente, soit exactement, soit à une constante multiplicative près.

Cette approche montre bien les similitudes entre les divers indices : elle met par exemple en évidence la parenté qui unit Banzhaf et Holler-Packel. Elle peut en outre suggérer la construction de nouveaux indices, soit par des croisements inédits des réponses proposées à chacune des deux questions, soit en introduisant de nouvelles réponses. On peut ainsi considérer, dans le cadre d'une formalisation des processus de décision parlementaires, que les seules coalitions admissibles sont celles qui sont formées par des partis proches sur le plan idéologique (coalitions dites « connectées $\gg)^{15}$. On obtient ainsi une nouvelle famille de coalitions admissibles, à laquelle on peut associer divers indices de pouvoir en fonction de la réponse choisie à la seconde question.

\section{PREMIERS ÉLÉMENTS DE COMPARAISON}

D'un point de vue empirique, la façon la plus simple et la plus immédiate de comparer les différents indices que nous avons présentés consiste à les appliquer à un même exemple, si possible réel. Afin d'illustrer cette démarche, nous considérons le jeu de vote du Conseil de l'Union européenne. Dans sa première version de 1958, ce Conseil était constitué de six membres : Allemagne, France, Italie, Belgique, PaysBas et Luxembourg. Les trois premiers disposaient de 4 voix, les deux suivants de 2 voix et le Luxembourg avait une voix unique pour un total de 17 voix. Les décisions étaient prises avec une règle de majorité qualifiée : toute proposition devait obtenir au moins 12 voix sur 17 pour être adoptée. Formellement, ce jeu de vote pondéré s'écrit $v$ : $[12 ; 4,4,4,2,2,1]$. Nous avons appliqué les différents indices introduits plus haut à ce jeu pondéré. Les résultats sont présentés dans le Tableau $4^{16}$. Dans ce tableau, le joueur 1 correspond à un membre du Conseil ayant 4 voix, tandis que les joueurs 2 et 3 correspondent aux membres du Conseil ayant respectivement 2 voix et 1 voix.

\footnotetext{
${ }^{15}$ Les contributions récentes de Edelman [1997], Perlinger [2000] et Bilbao [2000] relèvent de cette démarche.

${ }^{16}$ Afin de faciliter la comparaison, nous ne présentons que la version normalisée de chacun des indices.
} 
Tableau 4

\begin{tabular}{|c|c|c|c|}
\hline & Joueur 1 & Joueur 2 & Joueur 3 \\
\hline$S S$ & 0,2333 & 0,15 & 0 \\
\hline$\tilde{B}$ & 0,2381 & 0,1429 & 0 \\
\hline$\tilde{J}$ & 0,25 & 0,125 & 0 \\
\hline$D P$ & 0,2083 & 0,1875 & 0 \\
\hline$\tilde{H P}$ & 0,2 & 0,2 & 0 \\
\hline$\tilde{C M}$ & 0,25 & 0,125 & 0 \\
\hline$\tilde{A B}$ & 0,2420 & 0,1371 & 0 \\
\hline$C$ & 0,2778 & 0,0856 & 0 \\
\hline
\end{tabular}

En dépit de différences numériques, les divers indices considérés conduisent à des conclusions globalement similaires. Ils indiquent que la part de pouvoir d'un joueur de type 1 est comprise entre $20 \%$ et $28 \%$ et que celle d'un joueur de type 2 est comprise entre $8,5 \%$ et $20 \%$ (l'indice de Holler-Packel se distingue cependant en donnant le même poids à un joueur de type 1 et à un joueur de type 2). Les divers indices s'accordent par ailleurs à donner un pouvoir nul au Luxembourg : que ce pays vote pour ou contre une proposition n'a aucune incidence sur le résultat final.

Ces premiers résultats sont-ils de nature à suggérer que la question du choix de la mesure de pouvoir la plus appropriée pourrait être, sinon sans objet, du moins d'une importance secondaire? Nous allons voir qu'une telle conclusion serait certainement erronée. Pour cela, considérons tout d'abord le même Conseil européen mais en supposant que la règle de décision change et devienne une règle de majorité simple : une proposition doit obtenir 9 voix sur 17 pour être adoptée. Le jeu pondéré considéré est donc le suivant : $v:[9 ; 4,4,4,2,2,1]$. Le Tableau 5 présente les résultats des calculs des indices de pouvoir.

Tableau 5

\begin{tabular}{|c|c|c|c|}
\hline & Joueur 1 & Joueur 2 & Joueur 3 \\
\hline$S S$ & 0,2333 & 0,1 & 0,1 \\
\hline$\tilde{B}$ & 0,2333 & 0,1 & 0,1 \\
\hline$\tilde{J}$ & 0,2538 & 0,0795 & 0,0795 \\
\hline$D P$ & 0,1987 & 0,1346 & 0,1346 \\
\hline$\tilde{H P}$ & 0,1905 & 0,1429 & 0,1429 \\
\hline$\tilde{C M}$ & 0,1987 & 0,0952 & 0,0476 \\
\hline$\tilde{A B}$ & 0,2333 & 0,1 & 0,1 \\
\hline$C$ & 0,2828 & 0,0606 & 0,0303 \\
\hline
\end{tabular}

On constate que, bien que les poids soient demeurés inchangés, la modification du quota a provoqué d'importants bouleversements dans les mesures de pouvoir. 
D'une part, tous les indices montrent que le pouvoir du Luxembourg est devenu non nul. D'autre part, les indices divergent pour mesurer le pouvoir relatif entre les pays de poids respectifs 2 et 1 : les indices de Colomer-Martinez et Chakravarty indiquent un pouvoir relatif identique à celui des poids (les pays de poids 2 ont un pouvoir relatif qui est le double de celui des pays de poids 1) tandis que les autres indices suggèrent que ces deux groupes de pays ont le même pouvoir. Un joueur de type 2 a-t-il oui on non plus de pouvoir que le joueur de type 3 ? Il devient difficile de répondre à cette question.

Les différences de mesure de pouvoir entre les indices sont ici plus significatives et le problème du choix de l'indice de pouvoir le plus approprié se trouve clairement posé. Cet exemple illustre en outre l'intérêt que peut avoir l'usage des indices pour mesurer l'impact d'un changement de règle de vote sur la répartition des pouvoirs entre les joueurs.

Considérons à présent le Conseil actuel de l'Union européenne. Ce Conseil est constitué de quinze membres : l'Allemagne, la France, la Grande-bretagne et l'Italie disposent de 10 voix, l'Espagne de 8 voix, la Belgique, la Grèce, les Pays-Bas et le Portugal de 5 voix, l'Autriche et la Suède de 4 voix, le Danemark, la Finlande et l'Irlande de 3 voix et le Luxembourg de 2 voix, soit un total de 87 voix. Toute proposition doit obtenir au moins 62 voix sur les 87 pour être adoptée. Le jeu pondéré est donc le suivant : $v:[62 ; 10,10,10,10,8,5,5,5,5,4,4,3,3,3,2]$. Les résultats des calculs des indices de pouvoir normalisés sont présentés dans le Tableau 6. Dans ce tableau, les joueurs 1, 2, 3, 4, 5 et 6 représentent les membres du Conseil ayant respectivement dix, huit, cinq, quatre, trois et deux voix.

Tableau 6

\begin{tabular}{|c|c|c|c|c|c|c|}
\hline & Joueur 1 & Joueur 2 & Joueur 3 & Joueur 4 & Joueur 5 & Joueur 6 \\
\hline$S S$ & 0,1167 & 0,0955 & 0,0552 & 0,0454 & 0,0353 & 0,0207 \\
\hline$\tilde{B}$ & 0,1116 & 0,0924 & 0,0587 & 0,0479 & 0,0359 & 0,0226 \\
\hline$\tilde{J}$ & 0,1330 & 0,1001 & 0,0490 & 0,0377 & 0,0267 & 0,0167 \\
\hline$D P$ & 0,0822 & 0,0751 & 0,0647 & 0,0608 & 0,0572 & 0,0440 \\
\hline$\tilde{H P}$ & 0,0809 & 0,0743 & 0,0650 & 0,0613 & 0,0582 & 0,0450 \\
\hline$\tilde{C M}$ & 0,1297 & 0,0953 & 0,0522 & 0,0393 & 0,0280 & 0,0144 \\
\hline$\tilde{A B}$ & 0,1114 & 0,0925 & 0,0589 & 0,0480 & 0,0358 & 0,0228 \\
\hline$C$ & 0,1565 & 0,1037 & 0,0412 & 0,0269 & 0,0151 & 0,0063 \\
\hline
\end{tabular}

Comme pour le Conseil des six, la comparaison cardinale n'apporte pas d'éléments déterminants dans la mesure où tous les indices considérés proposent une hiérarchie identique du pouvoir, correspondant à la hiérarchie des poids. On notera cependant que les écarts de pouvoir entre grands et petits pays (entre joueur 1 et joueur 6) sont minimisés par les mesures de Deegan-Packel et Holler-Packel et maximisés par l'indice de Chakravarty ${ }^{17}$.

\footnotetext{
${ }^{17}$ D'une manière générale, les calculs effectués dans cette section mettent en évidence deux tendances conformes à l'intuition : d'une part la considération des seules coalitions minimales
} 
Que se passe-t-il si l'on substitue un seuil de $50 \%$ (44 votes sur 87 ) à la régle de majorité qualifiée qui prévaut actuellement? Le Tableau 7 , qui prend en compte le jeu $v$ : $[44 ; 10,10,10,10,8,5,5,5,5,4,4,3,3,3,2]$, permet de répondre à cette question.

Tableau 7

\begin{tabular}{|c|c|c|c|c|c|c|}
\hline & Joueur 1 & Joueur 2 & Joueur 3 & Joueur 4 & Joueur 5 & Joueur 6 \\
\hline$S S$ & 0,1183 & 0,0917 & 0,0556 & 0,0464 & 0,0326 & 0,0218 \\
\hline$\tilde{B}$ & 0,1172 & 0,0914 & 0,0561 & 0,0468 & 0,0332 & 0,0220 \\
\hline$\tilde{J}$ & 0,1484 & 0,0983 & 0,0415 & 0,0324 & 0,0212 & 0,0137 \\
\hline$D P$ & 0,0732 & 0,0730 & 0,0674 & 0,0654 & 0,0617 & 0,0486 \\
\hline$\tilde{H P}$ & $\mathbf{0 , 0 7 0 3}$ & $\mathbf{0 , 0 7 1 2}$ & 0,0681 & 0,0665 & 0,0639 & 0,0509 \\
\hline$\tilde{C M}$ & 0,1181 & 0,0958 & 0,0572 & 0,0447 & 0,0322 & 0,0171 \\
\hline$\tilde{A B}$ & 0,1173 & 0,0914 & 0,0561 & 0,0468 & 0,0332 & 0,0220 \\
\hline$C$ & 0,1615 & 0,1007 & 0,0387 & 0,0258 & 0,0137 & 0,0061 \\
\hline
\end{tabular}

Les résultats obtenus dans les trois premiers cas suggéraient qu'il était possible d'établir une relation «naturelle » entre le poids et le pouvoir des membres du Conseil : à un poids supérieur correspondait un pouvoir égal ou supérieur. Ce dernier exemple montre qu'établir une telle relation n'est pas si évident. En effet, avec une règle de vote majoritaire, le membre du Conseil ayant huit voix (l'Espagne) a plus de pouvoir qu'un membre ayant dix voix lorsque l'on utilise l'indice de Holler-Packel. Les autres indices disent au contraire que le joueur de type 2 (l'Espagne) a moins de pouvoir que les joueurs de type 1. C'est donc ici la hiérarchie même du pouvoir qui est en cause.

Ainsi, à l'aide d'exemples ayant le mérite de ne pas être $a d$ hoc, il est possible de mettre en exergue la divergence des indices tant d'un point de vue cardinal qu'ordinal. Cette constatation ne fait que confirmer ce que nous enseigne la littérature théorique sur les indices de pouvoir. On sait depuis longtemps que les deux indices les plus connus, celui de Banzhaf et celui de Shapley-Shubik, sont asymptotiquement très différents (voir par exemple [Dubey et Shapley, 1979]). Sur le plan empirique, Straffin [1977] mentionne l'exemple du processus d'amendement de la constitution canadienne, dans lequel les indices de Shapley-Shubik et Banzhaf classent les provinces canadiennes de manière différente.

Comment peut-on alors effectuer un choix entre les différents indices qui nous sont proposés? Au-delà de la simple considération de leur formulation, quels critères peut-on utiliser pour les départager? Les deux sections qui suivent tentent d'apporter des éléments de réponse.

gagnantes dans le calcul d'un indice tend à réduire les écarts de pouvoir entre poids « lourds » et poids « légers »; d'autre part, la prise en compte des $w_{i}$ dans la formule de calcul de l'indice tend à augmenter ces écarts. 


\section{AXIOMES, POSTULATS ET PARADOXES}

\subsection{L'APPROCHE AXIOMATIQUE}

Un certain nombre d'indices ont été caractérisés comme étant les seuls à vérifier une liste réduite d'axiomes. Shapley et Shubik [1954] proposent ainsi une justification de nature essentiellement axiomatique de leur indice. L'indice de Banzhaf non normalisé a été axiomatisé par Owen [1978] et Dubey et Shapley [1979]. Des caractérisations alternatives de Banzhaf et Shapley-Shubik et des axiomatisations d'autres indices sont apparues par la suite. Le lecteur est renvoyé à Laruelle [1998] pour une présentation simple et rapide des principaux résultats.

En dépit de l'intérêt mathématique de certaines d'entre elles, ces axiomatisations sont malheureusement difficilement utilisables pour guider le choix d'un indice de pouvoir. Plusieurs raisons expliquent ce constat. Il convient tout d'abord de souligner que tous les indices présentés n'ont pas fait l'objet d'axiomatisations : les indices de Johnston, Colomer-Martinez et Andjiga-Berg n'ont pas, à notre connaissance, été caractérisés. On ne peut donc procéder à une comparaison générale des indices, à moins de considérer que les indices non caractérisés axiomatiquement doivent être rejetés. En deuxième lieu, deux indices peuvent avoir des caractérisations n'ayant aucun axiome en commun, ce qui ne facilite pas la comparaison. Par exemple, Barua et alii [2002] proposent une axiomatisation de l'indice de Chakravarty qui n'utilise aucun des axiomes habituels en théorie des jeux. Comment dès lors peut-on le comparer aux autres indices? Le troisième et dernier argument que l'on peut avancer - certainement le plus important - concerne l'absence de signification intuitive, en terme de pouvoir dans un processus de décision collective, de la plupart des axiomes proposés. Cette difficulté est clairement liée à la filiation des indices de pouvoir avec la théorie des jeux coopératifs : certains axiomes qui paraissent naturels en théorie des jeux n'ont plus d'interprétation évidente lorsqu'on se limite aux jeux simples et à l'analyse du pouvoir. En dehors de ceux que tous les indices vérifient, les axiomes qui apparaissent dans les résultats de caractérisation sont souvent difficilement interprétables et parfois même manifestement ad hoc. De nombreux exemples pourraient être fournis. Nous n'en donnerons qu'un, celui de l'axiome de composition, qui intervient dans la caractérisation de Shapley-Shubik et de Banzhaf. Afin d'énoncer cet axiome, nous introduisons deux notations supplémentaires : étant donné deux jeux simples $(N, v)$ et $(N, w)$, posons $(v \wedge w)(S)=\min (v(S), w(S))$ et $(v \vee w)(S)=\max (v(S), w(S))$. On a alors, en notant $\alpha_{i}(v)$ l'indice de pouvoir du joueur $i$ dans le jeu $v$.

AXiome De Composition. Soient $(N, v)$ et $(N, w)$ deux jeux simples. Alors :

$$
\forall i \in N, \quad \alpha_{i}(v \wedge w)+\alpha_{i}(v \vee w)=\alpha_{i}(v)+\alpha_{i}(w)
$$

L'intuition qui sous-tend cet énoncé est loin d'être évidente. Comme le note Straffin [1982], "if we try to think of how to interpret [the axiom] as a statement about power in political situations then the best we can say is that it appears "somewhat opaque'". Felsenthal et Machover [1995] vont plus loin : "It seems to us that this axiom was introduced on a posteriori grounds, in order to guarantee the 
uniqueness of the result, rather than on grounds of a priori plausibility". C'est ce type d'arguments qui a conduit Felsenthal et Machover [1995], [1998] à proposer une approche un peu différente pour évaluer les indices de pouvoir ${ }^{18}$.

\subsection{POSTULATS ET PARADOXES}

Afin d'éviter les écueils de l'analyse axiomatique, Felsenthal et Machover [1995], [1998] suggèrent de considérer des propriétés « naturelles », généralement appelées postulats, qu'un indice « raisonnable » devrait satisfaire. Le problème est alors de distinguer les indices qui vérifient ces propriétés de ceux qui ne les vérifient pas, la qualité d'un indice s'évaluant au nombre de propriétés qu'il vérifie. Le fait qu'une mesure de pouvoir viole un de ces postulats donne naissance à un paradoxe ${ }^{19}$. Bien qu'il existe une grande variété de paradoxes dans la littérature, nous n'en considérerons ici que trois. Il s'agit du paradoxe de la monotonie, du paradoxe du transfert et du paradoxe du bloc, qui sont tous les trois considérés par Felsenthal et Machover comme des «pathologies disqualifiantes ».

- Le paradoxe de la (non-)monotonie est fondé sur le postulat selon lequel plus le poids d'un individu est important dans un jeu simple pondéré, plus son pouvoir doit être important. Formellement, ce paradoxe peut s'énoncer de la manière suivante :

PARAdoXe De la monotonie. Soit $(N, v)$ un jeu simple pondéré, avec un quota $q$ et une distribution de poids $\left(w_{1}, \ldots, w_{n}\right)$. L'indice $\alpha$ est sujet au paradoxe de la monotonie s'il existe deux joueurs $i$ et $j$ tels que $w_{i}>w_{j}$ et $\alpha_{i}(v)<\alpha_{j}(v)$.

Nous avons pu constater à travers l'exemple étudié dans la section précédente que l'indice de Holler-Packel ne satisfait pas au postulat de monotonie. Ce défaut est partagé par d'autres indices. Berg et Lane [1997] montrent en effet que pour les jeux simples pondérés de la forme $v:[2 a+1 ; a, a, a-1,1,1, \ldots, 1,1]$ à $a+5$ joueurs, le nombre de coalitions minimales gagnantes auxquelles le joueur de poids $a-1$ appartient est plus grand que le nombre de coalitions minimales gagnantes auxquelles un joueur de poids $a$ appartient. Ce résultat suggère que les mesures de pouvoir fondées sur les coalitions minimales gagnantes sont susceptibles de donner lieu au paradoxe de la monotonie. Les indices de Shapley-Shubik, Banzhaf (normalisé ou non), Johnston, Andjiga-Berg et Chakravarty vérifient en revanche le postulat de monotonie.

\footnotetext{
${ }^{18}$ Notons que ces arguments ne disqualifient pas l'approche axiomatique. Ils indiquent simplement qu'il n'existe pas actuellement d'ensemble d'axiomes facilement interprétables susceptibles de caractériser et de comparer les divers indices proposés dans la littérature. Le récent travail de Laruelle et Valenciano [2001], qui proposent des axiomes «transparents en termes de pouvoir dans un processus de décision collective » pour caractériser les indices de Shapley-Shubik et Banzhaf, constitue un premier effort pour pallier cette carence.

${ }^{19}$ À l'inverse, la négation d'un paradoxe conduit à un postulat. C'est en fait de cette manière que Felsenthal et Machover [1995] définissent leurs postulats.
} 
- Le deuxième postulat que nous considérons indique qu'un individu ne devrait pas augmenter son pouvoir en donnant une partie de son poids à un autre individu. Le viol de ce postulat donne lieu au paradoxe du transfert qui peut s'énoncer de la faon suivante :

PARADOXE DU TRANSFERT. Soient $(N, v)$ un jeu simple pondéré avec un quota $q$ et une distribution de poids $\left(w_{1}, \ldots, w_{n}\right)$ et $\left(N, v^{\prime}\right)$ le jeu simple pondéré avec le même quota $q$ et une distribution de poids $\left(w_{1}^{\prime}, \ldots, w_{n}^{\prime}\right)$ telles que $\sum_{j \in N} w_{j}^{\prime}=\sum_{j \in N} w_{j}$. Supposons en outre qu'il existe $i$ vérifiant pour tout $j \neq i, w_{j}^{\prime} \geq w_{j}$ (et par conséquent $\left.w_{i}^{\prime}<w_{i}\right)$. L'indice $\alpha$ est sujet au paradoxe du transfert si $\alpha_{i}\left(v^{\prime}\right)>\alpha_{i}(v)$.

Afin d'illustrer ce paradoxe, considérons le jeu simple pondéré $v:[8 ; 5,3,1,1,1]$. Si nous mesurons le pouvoir des individus à l'aide de l'indice de Deegan-Packel, nous obtenons les résultats suivants :

$$
D P_{1}(v)=3 / 8, D P_{2}(v)=1 / 4 \text {, et pour } i \in\{3,4,5\}, D P_{i}(v)=1 / 8
$$

Considérons à présent le jeu simple pondéré $v^{\prime}$ : [8;4,4,1,1,1] obtenu à partir du jeu précédent en transférant une partie du poids de l'individu 1 à l'individu 2 . Pour ce jeu $v^{\prime}$, l'indice de Deegan-Packel nous donne les résultats suivants :

$$
D P_{1}\left(v^{\prime}\right)=D P_{2}\left(v^{\prime}\right)=1 / 2 \text {, et pour } i \in\{3,4,5\}, D P_{i}\left(v^{\prime}\right)=0 .
$$

Dans cet exemple, le simple fait que l'individu 1 donne une partie de son poids lui permet d'augmenter son pouvoir. L'indice de Deegan-Packel est donc sujet au paradoxe du transfert. Ce n'est pas le seul indice à souffrir de cette pathologie, comme l'indique le Tableau 8.

- Le dernier paradoxe considéré est le paradoxe du bloc. Dans l'exemple précédent, le fait que l'individu 1 donne une partie de son poids à l'individu 2 augmente le pouvoir des deux individus. Ceci suggère que ces deux individus ont tout intérêt à agir d'un commun accord, c'est-à-dire à former un bloc. En effet, si les deux individus décident d'agir ensemble et de voter toujours de la même manière, le bloc ainsi constitué peut se comporter comme un dictateur. De manière plus générale, le postulat découlant de cette notion de bloc suggère qu'un bloc formé par deux individus au moins doit avoir un pouvoir au moins aussi important que la somme des pouvoirs des individus qui composent ce bloc. La violation de cette propriété conduit au paradoxe du bloc, que nous pouvons énoncer de la manière suivante :

PARAdoxe Du BLOC. Soient $(N, v)$ un jeu simple pondéré avec un quota q et une distribution de poids $\left(w_{1}, \ldots, w_{n}\right)$ et $\left(N \backslash\{j\}, v^{\prime}\right)$ le jeu simple pondéré avec le même quota $q$ et une distribution de poids $\left(w_{1}^{\prime}, \ldots, w_{j-1}^{\prime}, w_{j+1}^{\prime}, \ldots, w_{n}^{\prime}\right)$ telle que pour tout $k \notin\{i, j\}, w_{k}^{\prime}=w_{k}$ et $w_{i}^{\prime}=w_{i}+w_{j}$. L'indice $\alpha$ est sujet au paradoxe du bloc si $\alpha_{j}(v)>0$ et $\alpha_{i^{\prime}}\left(v^{\prime}\right)<\alpha_{i}(v)$. 
Considérons le jeu simple pondéré $v$ : [25; 9, 9,7,1,1,1,1,1,1,1] afin d'illustrer ce paradoxe. Si la mesure de pouvoir utilisée est l'indice de Banzhaf normalisé, nous obtenons les résultats suivants :

$$
\begin{gathered}
\tilde{B}_{1}(v)=\tilde{B}_{2}(v)=129 / 392 \simeq 0,329, \tilde{B}_{3}(v)=127 / 392 \\
\quad \text { et pour } i \in\{4,5,6,7,8,9,10\}, \tilde{B}_{i}(v)=1 / 392
\end{gathered}
$$

Considérons, à présent, le jeu simple pondéré $v^{\prime}$ : [25; 10, 9, 7, 1, 1, 1, 1, 1, 1] obtenu à partir du jeu $v$ suite à la formation d'un bloc entre l'individu 1 et l'individu 10 . Pour ce jeu $v^{\prime}$, nous avons les résultats suivants :

$$
\begin{gathered}
\tilde{B}_{1}\left(v^{\prime}\right)=\tilde{B}_{2}\left(v^{\prime}\right)=65 / 199 \simeq 0,327, \tilde{B}_{3}\left(v^{\prime}\right)=63 / 199, \\
\text { et pour } i \in\{4,5,6,7,8,9\}, \tilde{B}_{i}\left(v^{\prime}\right)=1 / 199 .
\end{gathered}
$$

Ainsi, suite à la formation d'un bloc avec l'individu 10, l'individu 1 a un pouvoir moins important. L'indice de Banzhaf normalisé est donc sujet au paradoxe du bloc. Là encore, il n'est pas le seul à exhiber cette « anomalie ». Le Tableau 8 résume les propriétés des différents indices de pouvoir $^{20}$ (la présence d'un « oui » signifie que l'indice considéré vérifie le postulat associé au paradoxe éponyme).

Tableau 8

\begin{tabular}{|c|c|c|c|}
\hline & Bloc & Monotonie & Transfert \\
\hline$S S$ & oui & oui & oui \\
\hline$B$ & oui & oui & oui \\
\hline$\tilde{B}$ & non & oui & non \\
\hline$\tilde{J}$ & non & oui & non \\
\hline$D P$ & non & non & non \\
\hline$\tilde{H P}$ & non & non & non \\
\hline$\tilde{C M}$ & non & non & non \\
\hline$\tilde{A B}$ & non & oui & non \\
\hline$C$ & oui & oui & oui \\
\hline
\end{tabular}

Ces résultats appellent plusieurs commentaires. En premier lieu, le cas de l'indice de Banzhaf montre que la normalisation d'un indice, opération qui semble a priori naturelle, peut modifier considérablement ses propriétés. Il apparaît en second lieu que les indices de Shapley-Shubik, de Banzhaf non normalisé et de Chakravarty sont les seuls qui ne violent aucun des paradoxes considérés. D'autres propriétés sont donc nécessaires pour les discriminer. Il est sans doute quelque peu étonnant de trouver l'indice de Chakravarty au même rang que ceux de Shapley-Shubik et

\footnotetext{
${ }^{20}$ Les résultats relatifs aux indices de Shapley-Shubik, Banzhaf, Johnston, Deegan-Packel et Holler-Packel se trouvent dans Felsenthal et Machover [1995]; les résultats concernant l'indice de Chakravarty viennent de Chakravarty [2000]; la preuve des résultats relatifs aux indices de Colomer-Martinez et Andjiga-Berg est donnée en annexe.
} 
Banzhaf (non normalisé), dans la mesure où cet indice ne possède pas ou peu de fondement intuitif ni empirique et qu'il conduit (on l'a vu) à amplifier de manière peut-être exagérée les écarts de pouvoir entre joueurs. Autrement dit, un indice à la plausibilité douteuse peut passer sans encombre le test des postulats, ce qui peut conduire à s'interroger sur la validité de la démarche.

Il faut savoir par ailleurs que les postulats ayant conduit aux paradoxes ci-dessus font l'objet de plusieurs controverses. Le postulat de monotonie, en particulier, a été vivement discuté (voir par exemple [Brams et Fishburn, 1995]). Ce postulat, comme d'ailleurs le postulat du bloc ou celui du transfert, exige une forme de corrélation positive entre le poids d'un joueur et la mesure de son pouvoir. Mais une telle exigence est-elle légitime? Après tout, toute la théorie des indices de pouvoir nous enseigne que poids et pouvoir sont deux notions distinctes et que le pouvoir d'un joueur peut être fort différent de son poids. On peut ainsi se demander si l'occurrence d'un paradoxe ne traduirait pas la complexité de la notion même de pouvoir plutôt que la manifestation du comportement pathologique d'un indice. Afin d'illustrer ce dernier point, considérons à nouveau le jeu $v:[8 ; 5,3,1,1,1]$ et le jeu $v^{\prime}:[8 ; 4,4,1,1,1]$ obtenu à partir du précédent par un transfert de poids de l'individu 1 à l'individu 2. Le passage de $v$ à $v^{\prime}$ entraîne en fait un bouleversement dans la structure fondamentale du jeu, en modifiant complètement les coalitions gagnantes et les coalitions gagnantes minimales; les joueurs 3,4 et 5 qui avaient un pouvoir de décision dans $v$ deviennent des joueurs "nuls" dans $v^{\prime}$ et les joueurs 1 et 2 deviennent par conséquent les seuls joueurs décisifs. Il n'est donc pas "anormal" que le joueur 1 voie son pouvoir augmenté dans le passage de $v$ à $v^{\prime}$. Condamner un indice qui met en évidence cette augmentation de pouvoir est sans doute une attitude trop radicale. D'autres approches sont nécessaires pour comprendre et comparer les indices de pouvoir.

\section{INTERPRÉTATIONS PROBABILISTES}

\subsection{OBSERVATIONS PRÉLIMINAIRES}

Certains des indices que nous avons présentés sont explicitement définis en termes probabilistes (Curiel) ou font appel à une hypothèse probabiliste explicite (AndjigaBerg, Deegan-Packel). En fait, tous ces indices peuvent faire l'objet d'une interprétation probabiliste plus ou moins immédiate, qui consiste soit à les faire apparaître comme la probabilité qu'un joueur soit décisif, soit à les interpréter comme une espérance mathématique.

Compte tenu du caractère (en général) a priori des indices de pouvoir que l'on cherche à construire, il est naturel d'avoir recours à une forme d'équiprobabilité (l'indice de Curiel, qui autorise d'autres distributions, constitue de ce point de vue une exception). Cette hypothèse d'équiprobabilité porte en général sur des ensembles de coalitions, dont la définition varie d'un indice à l'autre, d'un auteur à l'autre. Il peut s'agir (la liste n'est pas exhaustive) :

(H1) de l'ensemble de toutes les coalitions qui peuvent se former (qui sont en nombre égal à $2^{n}$ si l'on accepte l'ensemble vide);

(H2) de l'ensemble de toutes les coalitions de taille $n$ avec prise en compte de l'ordre 
des joueurs qui les constituent (il y a $n$ ! « coalitions » de ce type) ;

(H3) de l'ensemble des coalitions auxquelles le joueur $i$ est susceptible d'appartenir (en nombre égal à $2^{n-1}$ );

(H4) de l'ensemble des coalitions gagnantes (on notera que le nombre de ces coalitions est $2^{n-1}$ lorsque le jeu considéré est propre et fort);

(H5) de l'ensemble des coalitions minimales gagnantes.

À l'aide de l'une ou l'autre de ces hypothèses, on peut alors interpréter certains des indices mentionnés plus haut comme la probabilité pour un joueur d'exercer une influence décisive sur le résultat du vote. Traditionnellement, l'indice de Banzhaf (non normalisé) du joueur $i$ s'interprète comme la probabilité sous l'hypothèse H3 que le joueur $i$ transforme une coalition gagnante en coalition perdante. Cet indice peut aussi être déduit de l'hypothèse H4 (si l'on considère que seules des coalitions gagnantes se formeront et que le jeu est décisif) ou de l'hypothèse H1 (en définissant le pouvoir comme la probabilité de transformer une coalition gagnante en coalition perdante ou de transformer une coalition perdante en coalition gagnante) ${ }^{21}$. Les indices d'Holler-Packel et de Shapley-Shubik se déduisent aisément quant à eux des hypothèses $\mathrm{H} 5$ et $\mathrm{H} 2$ (respectivement). On notera dès à présent que l'indice de ShapleyShubik peut recevoir une interprétation probabiliste complètement indépendante de toute notion d'ordre des joueurs. En effet, cet indice peut aussi s'analyser comme la probabilité pour qu'un joueur soit décisif (i.e. ait la capacité de transformer une coalition gagnante en coalition perdante), sachant que toutes les coalitions de taille $s$ auxquelles ce joueur est susceptible d'appartenir sont équiprobables (il y en a $\left.\frac{(n-1) !}{(s-1) !(n-s) !}\right)$ et que toutes les tailles de coalition $(s \in\{1,2, \ldots, n\})$ contenant ledit joueur sont équiprobables; la probabilité d'occurrence d'une coalition $S$ est alors égale à $\frac{(s-1) !(n-s) !}{(n-1) !} \frac{1}{n}=\frac{(s-1) !(n-s) !}{n !}$.

Les hypothèses probabilistes sur les divers (sous-)ensembles de coalitions peuvent aussi être utilisées pour exprimer les indices comme des espérances mathématiques de «gain ». Cette approche a l'avantage d'offrir une interprétation immédiate à tous les indices pour lesquels les joueurs perçoivent un «montant » de pouvoir différent de 1 dans les coalitions gagnantes où ils sont décisifs (pour ces indices, l'interprétation en terme de probabilité d'être décisif ne tient plus). Ainsi, les indices de Johnston, Deegan-Packel, Andjiga, Colomer et Chakravarty peuvent-ils s'interpréter de cette manière. Par exemple, l'indice de Deegan-Packel représente l'espérance mathématique de «pouvoir »du joueur $i$ lorsque chacun des joueurs reçoit un montant $1 / s$ quand il est décisif et que l'hypothèse H5 prévaut. Soulignons cependant que ce genre d'analyse n'apporte pas grand-chose de plus à la définition des indices et peut être contesté en ce qu'il se réfère à une notion de partage des gains qui reste assez obscure dans le cadre des jeux simples.

\subsection{L'ANAlyse DE Straffin}

Straffin [1977], s'inspirant d'Owen [1972], fut le premier à proposer une analyse probabiliste non triviale des deux indices de pouvoir les plus connus, à savoir l'indice

\footnotetext{
${ }^{21}$ Voir sur ce point l'article de Laruelle et Valenciano [2001].
} 
de Banzhaf et l'indice de Shapley-Shubik. Straffin s'interroge sur la probabilité que le vote d'un individu particulier affecte le résultat final lorsqu'une proposition est soumise au vote de l'ensemble des joueurs. Il introduit pour chaque proposition un vecteur d'acceptabilité $\left(p_{1}, \ldots, p_{n}\right)$, où $0 \leq p_{i} \leq 1$ désigne la probabilité que l'individu $i$ vote en faveur de la proposition. Compte tenu de l'absence d'information que suppose la construction d'un indice de pouvoir a priori, deux hypotèses alternatives lui paraissent alors naturelles.

Hypothèse d'indépendance: Chaque $p_{i}$ est choisi de manière indépendante dans une distribution uniforme sur $[0,1]$.

Hypothèse d'homogénéité: Un nombre $p$ est choisi dans la distribution uniforme $[0,1]$ et l'on pose $p_{i}=p$ pour tout $i$.

Notons $\alpha_{i}$ la probabilité que l'individu $i$ affecte le résultat final du vote. Straffin obtient le résultat suivant.

PROPOSITION 1 Straffin [1977]. La probabilité $\alpha_{i}$ est égale à $B_{i}$ sous l'hypothèse d'indépendance et à $S S_{i}$ sous l'hypothèse d'homogénéité.

Autrement dit, l'hypothèse d'homogénéité conduit à l'indice de Shapley-Shubik et l'hypothèse d'indépendance à l'indice de Banzhaf non normalisé. Ce résultat s'établit facilement. Pour tout élément $S$ de $\mathcal{D}_{i}$ (l'ensemble des coalitions gagnantes dans lesquelles le joueur $i$ est décisif), tous les membres de $S$ autres que $i$ votent pour la proposition et tous les joueurs qui ne sont pas dans $S$ votent contre. La probabilité que cela survienne peut s'écrire :

$$
h_{i}\left(p_{1}, \ldots, p_{n}\right)=\sum_{S \in \mathcal{G}_{i}} \prod_{j \in S-\{i\}} p_{j} \prod_{k \in N-S}\left(1-p_{k}\right) .
$$

Sous l'hypothèse d'indépendance, le pouvoir du joueur $i$, défini comme sa probabilité d'être décisif, est alors

$$
\begin{gathered}
\int_{0}^{1} \ldots \int_{0}^{1} h_{i}\left(p_{1}, \ldots, p_{n}\right) d p_{1} \ldots d p_{n} \\
=\sum_{S \in \mathcal{G}_{i}} \prod_{j \in S-\{i\}} \int_{0}^{1} p_{j} d p_{j} \prod_{k \in N-S} \int_{0}^{1}\left(1-p_{k}\right) d p_{k}=\sum_{S \in \mathcal{G}_{i}} \frac{1}{2^{n-1}}=B_{i} .
\end{gathered}
$$

Sous l'hypothèse d'homogénéité, le pouvoir du joueur $i$ s'écrit :

$$
\int_{0}^{1} h_{i}(p, \ldots, p)=\sum_{S \in \mathcal{G}_{i}} \int_{0}^{1} p^{s-1}(1-p)^{n-s} d p=\sum_{S \in \mathcal{G}_{i}} \frac{(s-1) !(n-s) !}{n !}=S S_{i} .
$$

Par conséquent, les deux indices peuvent être déduits d'un même modèle probabiliste et ce n'est pas tant la distinction « illusoire » (selon le qualificatif utilisé par Straffin) entre les notions de combinaison et de permutation qui doit guider le 
choix de l'un ou de l'autre ${ }^{22}$, mais bien plutôt le degré d'indépendance statistique entre les votants. Si l'on pense que ceux-ci font leurs choix de manière complètement indépendante, alors il faut utiliser l'indice de Banzhaf pour calculer leur pouvoir. Si, en revanche, on estime qu'ils jugent les différentes propositions qui leur sont soumises en fonction de valeurs communes, alors c'est l'indice de Shapley-Shubik qui apparaît comme le plus approprié.

Remarque 1. Straffin fait observer que les assemblées de votants sont dans bien des cas constituées de groupes se caractérisant par un comportement de vote qui est à la fois homogène à l'intérieur de chaque groupe et indépendant entre les groupes. Pour prendre en compte ce type de situations, il introduit une hypothèse dite d'homogénéité partielle, le conduisant à un indice combinant les caractéristiques de Banzhaf à celles de Shapley-Shubik. Nous renvoyons à l'article original de Straffin [1977] pour une présentation de cet indice et une intéressante application à la Constitution canadienne.

Remarque 2. Leech [1990] note que l'indice de Banzhaf peut être obtenu selon une analyse similaire à celle de Straffin sans qu'il soit nécessaire de supposer que tous les $p_{i}$ sont issus d'une loi uniforme sur $[0,1]$ : il suffit en fait de supposer que les $p_{i}$ sont sélectionnés (de manière indépendante) dans n'importe quel ensemble de distributions définies sur $[0,1]$ ayant pour moyenne commune $1 / 2$. Leech en conclut que les hypothèses probabilistes qui sous-tendent l'indice de Banzhaf sont moins contraignantes que celles qui fondent l'indice de Shapley-Shubik.

Remarque 3. Posons $f_{i}(p)=h_{i}(p, \ldots, p)$. On peut noter, suivant Owen [1972], que l'on a $B_{i}=f_{i}(1 / 2)$ et $S S_{i}=\int_{0}^{1} f_{i}(p) d p$. L'indice de Banzhaf peut donc tre obtenu en posant que la probabilité qu'un joueur vote "oui" est $p=1 / 2$. Il est clair que si l'on choisit $p=1 / 4$ ou $p=2 / 3$, l'on peut obtenir en calculant $f_{i}(p)$ une mesure, voire une hiérarchie des pouvoirs fort différente de celle que l'on obtient pour $p=1 / 2$. Cette remarque suggère de calculer la mesure du pouvoir de vote comme une moyenne des valeurs obtenues pour les différents $p$ possibles. C'est exactement ce que réalise l'indice de Shapley-Shubik, qui ne privilégie pas une valeur particulière de $p$ et qui peut, de ce point de vue, s'interpréter comme l'espérance mathématique de l'indice de Banzhaf généralisé $f_{i}(p)$. On peut voir dans cette interprétation un argument en faveur de l'indice de Shapley-Shubik.

\subsection{Un PROLONGEMENT : L'APPROCHE DE BRUCKNER}

Selon Bruckner [2001], tous les indices de pouvoir proposés dans la littérature peuvent s'écrire de la manière suivante :

\footnotetext{
${ }^{22}$ L'indice de Shapley-Shubik a souvent été rejeté au motif que le processus de formation des coalitions - tenant compte de l'ordre d'arrivée des votants - qui sous-tend sa définition n'est pas jugé réaliste dans beaucoup d'applications.
} 


$$
\alpha_{i}=\sum_{S_{i}} P\left(S_{i}\right) K_{i}\left(S_{i}\right)
$$

où $K_{i}\left(S_{i}\right)$ désigne la « contribution » du joueur considéré à chacune des coalitions à laquelle il appartient $\left(S_{i}\right)$ et $P\left(S_{i}\right)$ s'interprète comme la probabilité de la coalition $S_{i}$. Étant donné une proposition, la probabilité que le joueur $i$ vote en faveur de cette proposition est notée $p_{i}$. Suivant Straffin [1977], Bruckner considère trois hypothèses concernant les probabilités $p_{i}$ : l'hypothèse d'indépendance, l'hypothèse d'homogénéité et l'hypothèse d'homogénéité partielle (que nous n'envisagerons pas ici). Il est clair que l'on obtient

$$
P\left(S_{i}\right)=(1 / 2)^{n-1}
$$

sous l'hypothèse d'indépendance, et l'hypothèse d'homogénéité conduit à

$$
P\left(S_{i}\right)=\frac{\left(s_{i}-1\right) !\left(n-s_{i}\right) !}{n !},
$$

$s_{i}$ désignant le cardinal de $S_{i}$. Bruckner envisage ensuite deux spécifications du terme $K_{i}\left(S_{i}\right)$, qu'il identifie au «pouvoir » du joueur dans la coalition $S_{i}$. La première spécification considère la capacité du joueur à transformer une coalition gagnante en coalition perdante :

$$
K_{i}\left(S_{i}\right)=\left\{\begin{array}{lll}
1 & \text { si } S_{i} \in \mathcal{G}_{i} \\
0 & \text { autrement. }
\end{array}\right.
$$

La seconde spécification est similaire mais considère que seules les coalitions minimales gagnantes se formeront, ce qui selon Bruckner correspond aux cas où la décision collective porte sur un bien public :

$$
K_{i}\left(S_{i}\right)=\left\{\begin{array}{lll}
1 & \text { si } S_{i} \in \mathcal{M}_{i} \\
0 & \text { autrement. }
\end{array}\right.
$$

L'équation (1), jointe à l'hypothèse d'indépendance et à la relation (2), donne l'indice de Banzhaf; l'indice de Shapley-Shubik est obtenu de la même façon en remplaçant l'indépendance par l'homogénéité. À l'aide des relations (1) et (3) et de l'hypothèse d'indépendance, Bruckner obtient un indice qui, une fois normalisé, coïncide avec l'indice normalisé de Holler-Packel. Il propose en outre deux nouveaux indices, fondés sur les relations (1), (3) et sur les hypothèses d'homogénéité et d'homogénéité partielle.

Il est clair que l'on peut facilement, en suivant l'analyse de Bruckner, proposer des spécifications de $K_{i}\left(S_{i}\right)$ qui, combinées à l'hypothèse d'indépendance, conduisent aux divers indices que nous avons présentés et qu'il ne considère pas dans son étude (Deegan-Packel, Johnston, Colomer-Martinez, Andjiga-Berg et Chakravarty). En dépit de son caractère ad hoc, cette approche prolonge utilement l'analyse de Straffin et, comme cette dernière, fournit un critère de sélection relativement objectif. Ainsi, comme le suggère Bruckner, les indices fondés sur l'hypothèse d'homogénéité sontils appropriés dans le contexte - par exemple - d'institutions internationales dont 
les états membres sont bien intégrés. Pour des institutions plus hétérogènes, les indices qui reposent sur l'hypothèse d'indépendance semblent plus adéquats. Enfin, on pourra recourir à l'hypothèse d'homogénéité partielle pour mesurer le pouvoir des différents membres d'une institution divisée en blocs de pays plus ou moins bien intégrés.

\subsection{L'APPORT DE BERG}

Bien qu'elle ne concerne que le cas des jeux propres et forts, l'analyse présentée par Berg [1999] complète et généralise à certains égards celle de Straffin. Étant donné un jeu simple propre et fort à $n$ joueurs, Berg introduit une classe de distributions de probabilité de type Polya-Eggenberger, définie sur l'ensemble des différentes coalitions possibles de la manière suivante :

$$
p(S, \delta)=1^{[s, \delta]} 1^{[n-s, \delta]} / 2^{[n, \delta]},
$$

expression dans laquelle $A^{[x, \delta]}=A(A+\delta) \ldots(A+(x-1) \delta)$ est une factorielle ascendante et le paramètre $\delta$ est supposé supérieur ou égal à $-1 /(n-1)$. Cette famille de modèles a été largement utilisée en théorie des choix collectifs pour calculer la probabilité de divers paradoxes du vote (voir sur ce point [Berg et Lepelley, 1993]). Affecter la valeur 0 au paramètre $\delta$ conduit à l'hypothèse de Culture impartiale (associée aux initiales IC dans la littérature anglo-saxonne) et correspond à ce que Straffin appelle l'indépendance. Prendre $\delta=1$ amène l'hypothèse de Culture anonyme et impartiale (IAC), ce que Straffin nomme homogénéité. On peut illustrer la génèse de la famille de distributions probabilistes introduite par Berg de la manière suivante : considérons une urne contenant deux types de boules en quantités égales. Chaque votant choisit au hasard une boule dans cette urne (le type de boule tiré fixant sa préférence, pour ou contre la proposition) puis remet dans l'urne la boule tirée plus $\delta$ boules du type choisi ${ }^{23}$. La probabilité qu'un ensemble $S$ de joueurs vote en faveur de la proposition est alors donnée par la relation (4). Ainsi, plus le paramètre $\delta$ est élevé, plus les participants au choix collectif auront tendance à voter de manière identique.

Berg munit ensuite l'ensemble des coalitions gagnantes d'un jeu propre et fort générique de la (classe de) mesure(s) de probabilité suivante :

$$
p^{*}(S, \delta)=\left\{\begin{array}{l}
2 p(S, \delta) \text { si } S \in G \\
0 \text { autrement. }
\end{array}\right.
$$

Notons que l'on a bien, pour un jeu propre et fort, $\sum_{S \in G} p^{*}(S, \delta)=1$. On vérifie en outre les relations :

$$
p^{*}(S, 0)=2 \frac{1}{2^{n}}=\frac{1}{2^{n-1}}
$$

\footnotetext{
${ }^{23}$ Notons que dans le modèle considéré, $\delta$ peut prendre des valeurs non entières, éventuellement négatives. Berg pose dans son étude $-1 /(n-1) \leq \delta \leq 1$.
} 
et

$$
p^{*}(S, 1)=2 \frac{s !(n-s) !}{(n+1) !}
$$

Posons :

$$
\eta_{i}(S)=v(S)-v(S-\{i\})
$$

Berg définit alors une famille de mesures de pouvoir de la manière suivante :

$$
\alpha_{g, \delta}^{i}=E_{\delta}\left[g(S) \eta_{i}(S)\right]
$$

expression dans laquelle l'espérance mathématique est calculée conformément à la relation $(5)$ et $g(S)$ est un poids non négatif attribué à la coalition $S$. Pour déterminer un indice de pouvoir, il faut donc fixer la valeur de deux paramètres, $\delta$ et $g(S)$.

On obtient (à un facteur de proportionnalité près) l'indice de Banzhaf en prenant $\delta=0$ et $g(S)=1$, l'indice de Shapley-Shubik en prenant $\delta=1$ et $g(S)=1$, l'indice de Johnston en prenant $\delta=0$ et $g(S)=1 / d(S)$, et l'indice d'Andjiga-Berg en prenant $\delta=0$ et $g(S)=1 / s$.

Berg établit par ailleurs quelques résultats intéressants que nous résumons dans la proposition suivante.

PROPOSITION $2([\mathrm{Berg}, 5])$. (i) $E_{\delta}\left[\eta_{i}(S)\right]$ est une fonction décroissante de $\delta$ et l'on $a \sum_{i \in N} E_{\delta}\left[\eta_{i}(S)\right]=1$ si et seulement si $\delta=1$;

(ii) $E_{\delta=1}\left[\eta_{i}(S) / s\right]=2 S S_{i} /(n+1)$;

(iii) $A B_{i} / A B_{k} \approx B_{i} / B_{k}$.

La premier point nous indique que la classe d'indices de type Banzhaf-ShapleyShubik dans lesquels $g(S)$ est constant tend à donner à un joueur un pouvoir d'autant plus faible que le paramètre $\delta$ est élevé; en outre, le seul indice normalisé (ou « efficient ») dans cette classe est l'indice de Shapley-Shubik. Les points (ii) et (iii) montrent que, dans la classe d'indices définie par la relation (6), la prise en compte de la taille des coalitions n'apporte pas d'autres informations que ce qui est déjà contenu dans les indices de Shapley-Shubik et de Banzhaf. Ce type de résultat, même s'il se limite aux jeux propres et forts, nous semble particulièrement éclairant pour le choix de l'indice approprié.

\section{REMARQUES FINALES}

- Indices a priori vs indices a posteriori

Une distinction majeure en matière d'indices de pouvoir est certainement celle qui oppose indices a priori et indices a posteriori. Cette distinction, qui recoupe en grande partie l'opposition normatif-positif évoquée dans l'introduction, ou bien encore l'opposition théorique-empirique, est très présente dans la littérature récente 
et une tendance s'affirme qui conduit les chercheurs à tenter de définir des mesures empiriques susceptibles d'être utilisées à des fins descriptives. Il est clair en effet qu'en règle générale, les indices classiques sont des mesures a priori mieux adaptées aux problèmes de "design" institutionnel (détermination du nombre de votes à accorder à chaque intervenant, choix du seuil majoritaire) qu'à la description de la réalité du pouvoir dans une assemblée donnée. Certains auteurs comme Garrett et Tsebelis [1999] vont jusqu'à condamner l'utilisation des indices classiques, considérant qu'ils ne prennent en compte que la seule information fournie par la règle de vote et ignorent complètement les «préférences » des votants et les comportements qu'elles induisent. Comment tenir compte de ces préférences? Une première voie de recherche, tracée par Owen [1977], [1982] et Myerson [1977] et suivie notamment par Bilbao [2000], consiste à définir des indices qui prennent en compte non seulement le mécanisme de vote mais aussi les relations entre les votants, sous la forme d'une structure coalitionnelle pré-existante censée représenter la tendance de certains groupes à adopter un comportement similaire. Ce type d'approche a été assez peu emprunté dans les applications, compte tenu notamment de la complexité des calculs qu'il implique (voir cependant [Chantreuil, 1996]). L'autre approche possible est de nature probabiliste. L'article récent de Laruelle et Valenciano [2002] rend bien compte de cette démarche (à laquelle on peut rattacher l'analyse de $\mathrm{Cu}-$ riel [1987], présentée dans la Section 3$)^{24}$. Selon ces auteurs, toute situation de vote se caractérise par deux ingrédients : la règle de vote d'une part, le comportement des votants d'autre part. Lorsqu'une proposition est soumise au groupe de décideurs, la mise en oeuvre de la règle de vote débouche sur une configuration de vote, c'est-à-dire sur une liste de « oui » et de «non ». Sous l'hypothèse qu'il n'y a pas d'abstention, il y a $2^{n}$ configurations possibles. Pour tout $S \subseteq N$, la configuration $S$ désigne le résultat du vote associé au cas où tous les éléments de $S$ votent « oui » et les autres «non ». Le comportement des votants est quant à lui résumé via une distribution de probabilité notée $p$ définie sur l'ensemble des configurations possibles. Cette distribution $p$ rend compte notamment des éventuelles proximités dans les préférences des votants. Par exemple, si tous les membres d'un même parti dans une assemblée observent une stricte discipline de vote, on affectera une probabilité nulle aux configurations dans lesquelles deux membres distincts de ce parti votent de manière différente. Laruelle et Valenciano proposent alors de mesurer le pouvoir de décision du votant $i$ de la manière suivante :

$$
\alpha_{i}(p)=\sum_{S \in \mathcal{D}_{i}^{-}} p(S)+\sum_{S \in \mathcal{D}_{i}^{+}} p(S),
$$

$\mathcal{D}_{i}^{-}$désignant l'ensemble des configurations gagnantes dans lesquelles le retrait du votant $i$ rend ces configurations perdantes et $\mathcal{D}_{i}^{+}$l'ensemble des configurations perdantes que l'arrivée du votant $i$ rend gagnantes. Cette approche permet d'étudier

\footnotetext{
${ }^{24}$ Les modèles probabilistes de la section précédente relèvent aussi, dans une certaine mesure, de cette approche, puisqu'ils débouchent souvent sur la construction de nouveaux indices censés être plus « réalistes ». Leur principal objectif reste cependant de fournir une interprétation probabiliste d'indices existants et diffère en cela de celui de Laruelle et Valenciano ou de Curiel.
} 
le pouvoir de vote dans des situations de vote réelles, en s'appuyant sur l'observation des votes passés ou sur toutes autres données pour estimer la distribution de probabilité des différentes configurations. Dans ces conditions, la question du «meilleur » indice ne se pose plus puisque l'indice le plus approprié est évidemment celui qui utilise la distribution de probabilité qui décrit la réalité de la manière la plus pertinente. Si, maintenant, l'on adopte un point de vue normatif, les votants et leur comportement n'ont plus à être pris en compte ${ }^{25}$. On peut alors, pour appliquer la formule proposée par Laruelle et Valenciano, faire l'hypothèse que toutes les configurations sont également probables. Il est facile de voir que cette hypothèse conduit à l'indice (non normalisé) de Banzhaf, dont l'usage à des fins normatives se trouve ainsi légitimé.

\section{- I-pouvoir vs P-pouvoir}

Au coeur des débats actuels se trouve une autre distinction que nous ne pouvons passer sous silence. Introduite (explicitement) par Felsenthal et Machover [1998], elle oppose ce qu'ils appellent le « I-pouvoir » et le « P-pouvoir ». Le I-pouvoir est le pouvoir en tant qu'«influence » : il mesure la capacité d'un électeur à contrôler le résultat d'un vote. Le P-pouvoir est quant à lui le pouvoir en tant que « prix » ou espérance de gain : il mesure, pour un électeur, la part relative qu'il peut espérer obtenir $\mathrm{du}$ «butin » que la coalition gagnante s'approprie. Pour Felsenthal et Machover, cette distinction est essentielle car ces deux notions de pouvoir sont totalement différentes et il importe de savoir quel type de pouvoir l'on souhaite mesurer. Les comportements de vote diffèrent en effet selon que l'on considère l'une ou l'autre de ces conceptions. La notion de I-pouvoir implique un comportement «idéologique » (ou "policy seeking" pour utiliser une terminologie issue des sciences politiques) en ce sens que les votants cherchent à mettre en oeuvre une politique ou une proposition particulière, qui s'appliqueront à l'ensemble des électeurs. Le P-pouvoir repose sur un comportement de type "office seeking" : les votants recherchent avant tout un bénéfice personnel (quelle qu'en soit la nature, financière ou sociale), bénéfice résultant d'un partage avec les autres membres de la coalition gagnante (et avec eux seulement). Notons qu'en sciences politiques, c'est ce type de comportement qui permet de justifier le principe de taille de Riker et la seule considération des coalitions minimales gagnantes.

Autre différence importante aux yeux de Felsenthal et Machover : la notion de marchandage qui est au coeur du P-pouvoir (il s'agit de partager un gain) est absente du I-pouvoir, qui peut parfaitement s'exercer dans un processus de vote à bulletins secrets. Il en résulte que, si l'analyse du P-pouvoir relève à l'évidence de la théorie des jeux coopératifs, le I-pouvoir peut s'en affranchir complètement. Ainsi, la notion même de «coalition », qui véhicule une idée de coordination consciente, est-elle peu appropriée pour l'analyse du I-pouvoir. Sans doute peut-on voir dans cette observation l'origine d'une tendance, de plus en plus courante au cours de ces dernières années, consistant à présenter les indices de pouvoir en termes purement

\footnotetext{
${ }^{25}$ Laruelle et Valenciano citent Straffin [1988] : "The fairness of the structure we design should not depend on the particular voters who will fill positions in that structure. As John Rawls might put it, formal justice should be designed behind a veil of ignorance".
} 
probabilistes, sans utilisation du vocabulaire de la théorie des jeux ${ }^{26}$.

La distinction I-P est bien entendu de nature à éclairer le problème du choix d'un indice de pouvoir a priori. Felsenthal et Machover [1998] ont sur la question un point de vue très tranché. Si l'on s'en tient aux indices les plus classiques, leurs conclusions tiennent en trois points :

1) toutes les tentatives sérieuses de formaliser la notion de I-pouvoir débouchent inéluctablement sur l'indice de Banzhaf (non normalisé);

2) les indices de Shapley-Shubik et Deegan-Packel relèvent du P-pouvoir;

3) l'indice de Johnston est quant à lui un indice hybride (puisqu'il introduit une correction de type P-pouvoir à l'indice de Banzhaf) et il convient de le rejeter.

Sans doute peut-on contester certaines de ces conclusions ${ }^{27}$. Il reste que la distinction I-P a permis de clarifier le débat et d'affranchir, au moins partiellement, l'analyse des indices de pouvoir de la théorie des jeux coopératifs.

- Mesure ordinale vs mesure cardinale

Nous nous sommes jusqu'ici intéressés à la théorie numérique de la mesure du pouvoir dans un jeu simple pondéré en attribuant, selon diverses modalités, une « quantité de pouvoir de décision » à chacun des joueurs. Face aux nombreuses difficultés - dont nous avons rendu compte - que présente cette approche, on peut être tenté de conclure que le pouvoir est une notion trop complexe et trop imprécise pour se laisser enfermer dans une valeur numérique. Ce point de vue est notamment défendu par [Morriss, 43] qui considère qu'un classement des joueurs selon leur pouvoir peut être suffisant. Divers auteurs ([Allingham, 1975], [Taylor, 1995], [Taylor et Zwicker, 1999], [Diffo Lambo et Moulen 2001, 2000]) ont adopté cette approche ordinale. Taylor et Zwicker définissent ainsi, pour tout jeu simple, un préordre entre les joueurs appelé relation d'influence de la manière suivante :

- les joueurs $i$ et $j$ sont d'égale influence dans le jeu simple $(N, v)$ si $\forall S \subseteq N-\{i, j\}$, $v(S+i)=1 \Leftrightarrow v(S+j)=1$;

- $i$ est plus influent que $j$ dans $(N, v)$ si $(\forall S \subseteq N-\{i, j\}, v(S+j)=1 \Rightarrow v(S+i)=1)$ et $(\exists S \subseteq N-\{i, j\}, v(S+j)=0$ et $v(S+i)=1)$;

- $i$ est au moins aussi influent que $j$ dans $(N, v)$, noté $i \succeq j$ si $i$ et $j$ sont d'égale influence dans $(N, v)$ ou si $i$ est plus influent que $j$ dans $(N, v)$.

Ils montrent que si $(N, v)$ est un jeu simple propre, alors la relation $\succeq$ est toujours un préordre sur $N$; ils caractérisent en outre les jeux simples pour lesquels $\succeq$ est un préordre total. Diffo Lambo et Moulen [2002] ont montré pour leur part que la relation $\succeq$ est un préordre total si et seulement si les ordres induits par les indices de

\footnotetext{
${ }^{26}$ Le modèle de Laruelle et Valenciano [2002] présenté dans le paragraphe précédent illustre bien cette tendance.

${ }^{27}$ Si l'on voit bien pourquoi l'indice de Deegan-Packel relève du P-pouvoir, le classement de Shapley-Shubik dans cette catégorie est certainement plus discutable. Bien qu'originellement lié à la théorie des jeux coopératifs, cet indice peut fort bien (on l'a vu) recevoir une interprétation probabiliste le rattachant clairement à l'analyse du I-pouvoir. Quant à l'indice de Johnston, son caractère hybride n'est peut-être pas une raison suffisante pour le condamner car, comme le reconnaissent eux-mêmes Felsenthal et Machover[1998], "there are undoubtely many real-life situations in which voting behaviour is driven by the two motives" (I et P).
} 
Shapley-Shubik et de Banzhaf du jeu $(N, v)$ coïncident avec $\succeq$, reliant ainsi l'analyse ordinale aux indices numériques.

\section{ANNEXE}

Nous montrons que l'indice de Colomer-Martinez viole les trois postulats que nous avons présentés et que l'indice d'Andjiga-Berg viole les propriétés de Bloc et de Transfert mais vérifie celle de Monotonie.

\section{Bloc}

- Pour le jeu simple pondéré $v$ : [25;9,9,7,1,1,1,1,1,1,1], si nous mesurons le pouvoir des individus à l'aide de l'indice d'Andjiga-Berg, nous obtenons les résultats suivants (les valeurs de l'indice sont données dans l'ordre du poids des joueurs) :

\begin{tabular}{|l|l|l|l|l|l|l|l|l|l|}
\hline 0,330324 & 0,330324 & 0,32696 & 0,0017 & 0,0017 & 0,0017 & 0,0017 & 0,0017 & 0,0017 & 0,0017 \\
\hline
\end{tabular}

Considérons à présent le jeu simple pondéré $v$ : $[25 ; 10,9,7,1,1,1,1,1,1]$. L'indice d'Andjiga-Berg répartit le pouvoir entre les individus de la façon suivante :

\begin{tabular}{|l|l|l|l|l|l|l|l|l|}
\hline 0,328354 & 0,328354 & 0,32149 & 0,0036 & 0,0036 & 0,0036 & 0,0036 & 0,0036 & 0,0036 \\
\hline
\end{tabular}

En formant un bloc avec le joueur 10, le nouveau joueur 1 voit son pouvoir diminuer. L'indice d'Andjiga-Berg viole donc la propriété du bloc.

- Pour le jeu simple pondéré $v$ : [13; 10, 2, 2, 2, 1, 1, 1, 1], si nous mesurons le pouvoir des individus à l'aide de l'indice de Colomer et Martinez, nous obtenons les résultats suivants :

\begin{tabular}{|l|l|l|l|l|l|l|l|}
\hline 0,76 & 0,048 & 0,048 & 0,048 & 0,024 & 0,024 & 0,024 & 0,024 \\
\hline
\end{tabular}

Considérons maintenant le jeu simple pondéré $v$ : [13;10,3, 2, 2, 1, 1, 1]. L'indice de Colomer et Martinez répartit le pouvoir entre les individus de la façon suivante :

\begin{tabular}{|l|l|l|l|l|l|l|}
\hline 0,7627 & 0,0254 & 0,0677 & 0,0677 & 0,0254 & 0,0254 & 0,0254 \\
\hline
\end{tabular}

En formant un bloc avec le joueur 8, le nouveau joueur 2 voit son pouvoir diminuer. L'indice de Colomer et Martinez viole donc la propriété du bloc.

\section{Monotonie}

- Montrons que l'indice d'Andjiga-Berg satisfait au postulat de monotonie. Soient deux joueurs $i$ et $j$ tels que $w_{i} \geq w_{j}$. Considérons une coalition $S$ telle que $S \in D_{j}$. Si $i \in S$, alors il est clair que $S \in \mathcal{D}_{i}$. Supposons que l'on ait $i \notin S$. Dans ce cas, la coalition $(S-\{j\}) \cup\{i\}$ appartient à $\mathcal{D}_{i}$ et le cardinal de $(S-\{j\}) \cup\{i\}$ est égal 
à $s$. Autrement dit, pour toute coalition $S \in D_{j}$, il existe une coalition $T$ de même cardinal telle que $T \in \mathcal{D}_{i}$. On a par conséquent :

$$
\sum_{S \in D_{j}} \frac{1}{s} \leq \sum_{S \in \mathcal{D}_{i}} \frac{1}{s} .
$$

Il en résulte que $A B_{j} \leq A B_{i}$.

- Pour le jeu simple pondéré $v$ : $[11 ; 5,5,4,1,1,1,1,1]$, si nous mesurons le pouvoir des individus à l'aide de l'indice de Colomer et Martinez,nous obtenons les résultats suivants :

\begin{tabular}{|l|l|l|l|l|l|l|l|}
\hline 0,27681 & 0,27681 & 0,29065 & 0,03114 & 0,03114 & 0,03114 & 0,03114 & 0,03114 \\
\hline
\end{tabular}

Le joueur avec un poids de 4 a un pouvoir plus important que les joueurs ayant un poids de 5. L'indice de Colomer et Martinez est donc sujet au paradoxe de la monotonie.

Transfert

- Pour le jeu simple pondéré $v$ : $[8 ; 5,3,1,1,1]$, si nous mesurons le pouvoir des individus à l'aide de l'indice d'Andjiga-Berg, nous obtenons les résultats suivants :

$$
\begin{array}{|l|l|l|l|l|}
\hline 0,30 & 0,25 & 0,0277 & 0,0277 & 0,0277 \\
\hline
\end{array}
$$

Considérons à présent le jeu simple pondéré $v^{\prime}$ : [8;4,4,1,1,1]. L'indice d'AndjigaBerg répartit le pouvoir entre les individus de la façon suivante :

$$
\begin{array}{|l|l|l|l|l|}
\hline 0,5 & 0,5 & 0 & 0 & 0 \\
\hline
\end{array}
$$

L'indice d'Andjiga-Berg viole la propriété du transfert puisque le joueur 1 augmente son pouvoir en transférant une partie de son poids au joueur 2 .

- Pour le jeu pondéré $v$ : $[13 ; 10,3,2,1,1,1,1]$, l'indice de Colomer et Martinez répartit le pouvoir de la manière suivante :

\begin{tabular}{|l|l|l|l|l|l|l|}
\hline 0,7692 & 0,02564 & 0,06837 & 0,03418 & 0,03418 & 0,03418 & 0,03418 \\
\hline
\end{tabular}

Considérons à présent le jeu $v^{\prime}$ : $[13,10,2,2,2,1,1,1]$. Si nous mesurons le pouvoir des individus à l'aide de l'indice de Colomer et Martinez, nous obtenons les résultats suivants :

\begin{tabular}{|l|l|l|l|l|l|l|}
\hline 0,7558 & 0,05813 & 0,05813 & 0,05813 & 0,02325 & 0,02325 & 0,02325 \\
\hline
\end{tabular}

Lorsque le joueur 2 transfère une partie de son poids au joueur 4, son pouvoir augmente. L'indice de Colomer et Martinez est donc sujet au paradoxe du transfert. 
Remerciements. Les auteurs remercient vivement Olivier Hudry, Vincent Merlin et Bernard Monjardet pour leur aide et leurs suggestions.

\section{BIBLIOGRAPHIE}

[1] ALLINGHAM M.G., «Economic Power and Values of Games », Zeitschrift für Nationalökonomie 35, 1975, p. 293-299.

[2] ANDJIGA N.G., Bargaining Models of Values for TU-games, document de travail, 1996.

[3] BANZHAF J., «Weigthed Voting Doesn't Work : A Mathematical Analysis », Rutgers Law Review 19, 1965, p. 317-343.

[4] BARUA R., S. CHAKRAVARTY et S. ROY, Power in Weighted Majority Games, mimeo, 2002.

[5] BERG S., « On Voting Power Indices and a Class of Probability Distributions with Applications to EU Data », Group Decision and Negotiation 8, 1999, p. 17-31.

[6] BERG S. et J.E. LANE, Measurements of Voting Power : Individual and Systemic Properties, mimeo, 1997.

[7] BERG S. et D. LEPELLEY, « Note sur le calcul de la probabilité des paradoxes du vote », Mathématiques, Informatique et Sciences humaines 120, 1993, p. 33-48.

[8] BILBAO J.M., Cooperative Games on Combinatorial Structures, Kluwer Academic Publisher, 2000.

[9] BOBAY F., «La réforme du Conseil de l'Union européenne à partir de la théorie des jeux », Revue Française d'Économie 16, 2001, p. 3-61.

[10] BONNET J. et D. LEPELLEY, « Pouvoir de vote et intercommunalité : le cas des établissements publics de coopération intercommunale de la région Basse-

Normandie », in R. Le Duff, J.-P. Rigal et G. Schmidt (éds.), Démocratie et management local, Dalloz, 2001.

[11] BRAMS S.J. et P.C. FISHBURN, « When Size is a Liability? Bargaining Power in Minimal Winnaning Coalitions », Journal of Theoretical Politics 7, 1995, p. 301316.

[12] BRUCKNER M., « Extended Probabilistic Characterization of Power Indices », in M.J. Holler et G. Owen (eds), Power Indices and Coalition Formation, Kluwer Academic Publishers, 2001.

[13] CAULIER, J.-F., Analyse économique de la vie politique : une application des indices de pouvoir à la formation de coalitions au parlement belge, Mémoire de maîtrise, F.U.N.D.P., 2001.

[14] CHAKRAVARTY S.R., Measurement of Power in Weigthed Majority Games, mimeo, 2000. 
[15] CHANTREUIL F., Valeurs de jeux coopératifs, Thèse de doctorat, Université de Caen, 1996.

[16] COLEMAN J.S., Individual Interests and Collective Action, Cambridge University Press, 1986.

[17] COLOMER J.M., « Measuring Parliamentary Deviation », European Journal of Political Research 30, 1996, p. 87-101.

[18] COLOMER J.M. et F. MARTINEZ, « The Paradox of Coalition Trading », Journal of Theoretical Politics 7, 1995, p. 41-63.

[19] CURIEL I.J., mbox«A Class of Non-Normalized Power Indices for Simple Games », Mathematical Social Sciences 13, 1987, p. 141-152.

[20] DEEGAN J. et E.W. PACKEL, «A New Index of Power for Simple n-Person Games », International Journal of Game Theory 7, 1978, p. 113-123.

[21] DEMANGE G., « Commentaire de l'article de F. Bobay », Revue française d'économie 16, 2001, p. 63-72.

[22] DIFFO LAMBO L. et J. MOULEN, Théorie du vote, pouvoir, procédures et prévisions, Paris, Hermès, 2001.

[23] DIFFO LAMBO L. et J. MOULEN, « Quel pouvoir mesure-t-on dans un jeu de vote? » Mathématiques et Sciences humaines 152, 2000, p. 27-47.

[24] DUBEY P. et L.S. SHAPLEY, « Mathematical Properties of the Banzhaf Power Index », Mathematics of Operations Research 4, 1979, p. 99-131.

[25] EDELMAN P., «A Note on Voting », Mathematical Social Sciences 34, 1997, p. $37-50$.

[26] FALCK, Y., Les Indices de pouvoir et la problématique d'une mesure objective d'un concept flou, Mémoire de D.E.A., Université de Caen, 2002.

[27] FELSENTHAL D.S. et M. MACHOVER, « Postulates and Paradoxes of Relative Voting Power - A Critical Reappraisal », Theory and Decision 38, 1995, p. $195-229$.

[28] FELSENTHAL D.S. et M. MACHOVER, The Measurement of Voting Power : Theory and Practice, Problems and Paradoxes, Edward Elgar, 1998.

[29] FELSENTHAL D.S. et M. MACHOVER, Voting Power Measurement : A Story of Misreinvention, mimeo, 2002.

[30] GARRETT G. et G. TSEBELIS, « Why Resist the Temptation to Apply Power Indices to the European Union? », Journal of Theoretical Politics 11, 1999, p. 291308.

[31] HOLLER M.J., « Forming Coalitions and Measuring Voting Power », Political Studies 30, 1982, p. 262-271.

[32] HOLLER M.J. et G. OWEN (eds), Power Indices and Coalition Formation, Kluwer Academic Publishers, 2001. 
[33] HOLLER M.J. et E.W. PACKEL, « Power, Luck and the Right Index », Journal of Economics 43, 1983, p. 21-29.

[34] JOHNSTON R., « On the Measurement of power : Some Reactions to Laver », Environment and Planning 10, 1978, p. 907-914.

[35] JUNN R.S., « La politique de l'amendement des articles 23 et 27 de la charte des Nations Unies », Mathématiques et Sciences humaines 40, 1972, p. 19-24.

[36] LARUELlE A., Game Theoretical Analysis of Decision-Making Processes with Applications to the European Union, Thèse de doctorat, Université catholique de Louvain, 1998.

[37] LARUELLE A. et F. VALENCIANO, « Shapley-Shubik and Banzhaf Indices Revisited », Mathematics of Operations Research 26, 2001, p. 89-104.

[38] LARUELlE A. et F. VALENCIANO, A Probabilistic Re-foundation of Voting Power Measures, Discussion Paper 14/2001, Departamento de Economia Applicada IV, Bilbao, Basque Country University, 2001.

[39] LARUELlE A. et F. VALENCIANO, Assessment of Voting Situations : the Probabilistic Foundations, Discussion Paper 26/2002, Departamento de Economia Applicada IV, Bilbao, Basque Country University, 2002.

[40] LEECH D., 1990, « Power Indices and Probabilistic Voting Assumptions », Public Choice 66, 1990, p. 293-299.

[41] LEECH D., 2000, «An Empirical Comparison of the Performance of Classical Power Indices », Political Studies 50, 2000, p. 1-22.

[42] MONJARDET, B., « Note sur les pouvoirs de vote au Conseil de Sécurité », Mathématiques et Sciences humaines 40, 1972, p. 25-28.

[43] MORRISS P., Power - A Philosophical Analysis, Manchester University Press, 1987, (réédité en 2002).

[44] MYERSON R., «Graphs and Cooperation in Games », Mathematics of Operations Research 2, 1977, p. 225-229.

[45] OWEN G., « Multilinear Extension of Games », Management Science 18, 1972, p. 64-79.

[46] OWEN G., «Values of Games with a priori Unions », in R. Hein and O. Moeschlin (eds), Essays in Mathematical Economics and Game Theory, Springer Verlag, 1977.

[47] OWEN G., « Characterization of the Banzhaf-Coleman Index », SIAM Journal of Applied Mathematics 35, 1978, p. 315-327.

[48] OWEN G., «Modification of the Banzhaf-Coleman Index for Games with a prior Unions », in : M. J. Holler (ed.), Power, Voting and Voting Power, WürzburgVienna, Physica Verlag, 1982.

[49] PAJAlA A., T. MESKANEN et T. KAUSE, Voting Power Website, http ://powerslave.val.utu.fi, 2002. 
[50] PENROSE L.S., « The Elementary Statistics of Majority Voting », Journal of the Royal Statistical Society 109, 1946, p. 53-57.

[51] PERLINGER T., «Voting Power in an Ideological Spectrum : The MarkovPolya Index », Mathematical Social Sciences 40, 2000, p. 215-226.

[52] RAE D.W., « Decision Rules and Individual Values in Constitutional Choice », American Political Science Review 63, 1969, p. 40-56.

[53] RIKER W.H., The Theory of Political Coalitions, New Haven and London, Yale University Press, 1962.

[54] RIKER W.H., « The First Power Index », Social Choice and Welfare 3, 1986, p. 293-295.

[55] SHAPLEY L.S., «A Value for n-Person Games », in H.W. Kuhn and A.W. Tucker, Contributions to the Theory of Games II, Annals of Mathematics Studies 28, 1953, p. 307-317.

[56] SHAPLEY L.S. et M. SHUBIK, «A Method for Evaluating the Distribution of Power in a Committee System », American Political Science Review 48, 1954, p. 787792.

[57] STRAFFIN P.D., «Homogeneity, Independance and Power Indices », Public Choice 30, 1977, p. 107-118.

[58] STRAFFIN P.D., 1982, « Power Indices in Politics », in S.J. Brams, W.F. Lucas et P.D. Straffin (eds.), Political and Related Models, New York, Springer, 1982, p. 256-321.

[59] STRAFFIN P.D., «The Shapley-Shubik and Banzhaf Power Indices as Probabilities », in A.E. Roth (eds.), The Shapley Value : Essays in Honor of Lloyd S. Shapley, Cambridge, Cambridge University Press, 1988.

[60] TAYLOR A.D., Mathematics and Politics - Strategy, Voting, Power and Proof, Springer-Verla, 1995.

[61] TAYLOR, A. D. et W.S. ZWICKER, Simple Games, Princeton University Press, 1999. 